\title{
Smoothness Analysis of Nonlinear Subdivision Schemes of Homogeneous and Affine Invariant Type
}

\author{
Gang Xie and Thomas P.-Y. Yu
}

\begin{abstract}
Nonlinear subdivision schemes arise from, among other applications, nonlinear multiscale signal processing and shape preserving interpolation. For the univariate homogeneous subdivision operator $S: \ell(\mathbb{Z}) \rightarrow \ell(\mathbb{Z})$ we establish a set of commutation/recurrence relations which can be used to analyze the asymptotic decay rate of $\left\|\Delta^{r} S^{j} m\right\|_{\ell^{\infty}}, j=1,2, \ldots$, the latter in turn determines the convergence and Hölder regularity of $S$. We apply these results to prove that the critical Hölder regularity exponent of a nonlinear subdivision scheme based on median-interpolation is equal to that of an approximating linear subdivision scheme, resolving a conjecture by Donoho and Yu [16], [4].

We also consider a family of nonlinear but affine invariant subdivision operators based on interpolation-imputation of $p$-mean (of which median corresponds to the special case $p=1$ ) as well as general continuous $M$-estimators. We propose a linearization principle which, when applied to $p$-mean subdivision operators, leads to a family of linear subdivision schemes. Numerical evidence indicates that in at least many cases the critical smoothness of a $p$-mean subdivision scheme is the same as that of the corresponding linear scheme. This suggests a more coherent view of the result obtained in this paper.
\end{abstract}

\section{Introduction}

In a broad sense, subdivision is a method of taking coarsely described data and recursively generating (typically smooth) data at finer and finer resolution. It is used in curve and surface generation for geometric modeling applications, and is also well known to be connected to wavelet construction via the MRA framework. For ease of implementation and analysis, the subdivision process is usually chosen to be linear, but, motivated by different applications, nonlinear schemes have also been proposed, e.g., [7], [9], [5], [10], [3], [4], [13], [2].

Donoho and Yu proposed in [4], [16] a family of nonlinear pyramid transforms for robust removal of non-Gaussian noise; the construction there was later generalized in [13]. Underlying this host of nonlinear pyramid transforms is a class of nonlinear subdi-

Date received: October 2, 2003. Date revised: May 17, 2004. Date accepted: August 3, 2004. Communicated by Peter Oswald. Online publication: December 22, 2004.

AMS classification: 26A15, 26A16, 26A18, 41A05, 42C40.

Key words and phrases: Subdivision scheme, Nonlinear subdivision scheme, Nonlinear wavelet transform, Hölder smoothness, Homogeneous map, Real projective plane, Median, $M$-Estimator, Linearization. 
vision operators for which no general theory is available for studying their convergence, smoothness, and stability properties. Smoothness and stability properties are of importance in transform-based signal processing applications. We address smoothness in this paper. Typically after one applies a pyramid transform to a signal, the transform coefficients are either thresholded or quantized for the purpose of, say, noise suppression or compression; subsequently, the processed coefficients are inverted and a reconstructed signal is obtained. Such a thresholding or quantization procedure in transform domain introduces certain artifacts in the reconstructed signal; knowing that the subdivision scheme underlying the pyramid transform is smooth guarantees that these artifacts do not appear excessively irregular to the viewers of the reconstructed signal. For wavelet bases there is of course a well-known approximation theoretic view of the just-described importance of smoothness properties. For instance, the smoothness of the elements in a wavelet basis, typically determined by the smoothness of an underlying subdivision process, determines, together with the accuracy order of the subdivision scheme, the range of Besov scale the wavelet basis can furnish unconditional basis for.

We recall here the nonlinear subdivision operators based on interpolation-imputation of $M$-estimators constructed in [13]. Here and below, let $\sigma: \mathbb{R} \rightarrow \mathbb{R}$ be a convex function that satisfies $\min \sigma=\sigma(0)=0$. Recall from [13] that the $M$-estimator with respect to $\sigma$ of a function $f \in C(I)$ is defined by

$$
m(f ; I, \sigma):=\underset{m \in \mathbb{R}}{\arg \min } \int_{I} \sigma(f(x)-m) d x .
$$

For example, $m(f ; I,|\cdot|)=\operatorname{median}(f \mid I)$ and $m\left(f ; I,|\cdot|^{2}\right)=\operatorname{average}(f \mid I)=$ $\int_{I} f(x) d x /|I|$. More generally, for $1 \leq p<\infty$,

$$
m\left(f ; I,|\cdot|^{p}\right)=\text { "p-mean of } f \text { over interval } I "=\underset{m \in \mathbb{R}}{\arg \min }\|f-m\|_{p} .
$$

This definition can be extended to $p=\infty$ by noticing that

(1.2) $\underset{m \in \mathbb{R}}{\arg \min }\|f-m\|_{\infty} \quad$ is unique and equals $\quad\left(\max _{I} f+\min _{I} f\right) / 2$.

Let $L \geq 1$ and $d \geq 2$ be integers. For a given $y \in \ell(\mathbb{Z})$ and $h>0$, define $S_{\sigma, L, d ; h}(y) \in$ $\ell(\mathbb{Z})$ as follows:

1. Interpolation: For each $k \in \mathbb{Z}$, let $p_{k} \in \Pi_{2 L}$ be the unique polynomial [13] such that

$$
m\left(p_{k} ;[h(k+l), h(k+l+1)], \sigma\right)=y_{k+l}, \quad l=-L, \ldots, L .
$$

\section{Imputation:}

$$
\left(S_{\sigma, L, d ; h}(y)\right)_{d k+l}:=m\left(p_{k} ;[h(k+l / d), h(k+(l+1) / d)], \sigma\right), \quad 0 \leq l<d .
$$

Since $m(f ; K, \sigma)=m\left(f(T \cdot) ; T^{-1}(K), \sigma\right)$ for any invertible affine map $T, S_{\sigma, L, d ; h}$ is independent of $h$, thus we drop the subscript $h$ and write

$$
S_{\sigma, L, d}: \ell(\mathbb{Z}) \rightarrow \ell(\mathbb{Z}) .
$$


When $\sigma=|\cdot|^{p}$, we write, for simplicity,

$$
S_{\sigma, L, d}=S_{p, L, d}, \quad 1 \leq p<\infty .
$$

Then $S_{\infty, L, d}$ is also well defined: simply replace $m(\cdot ; I, \sigma)$ in $(1.3)-(1.4)$ by the nonlinear functional (1.2).

Under this notation, the median-interpolating subdivision operator $S$ considered in Section 4 is $S_{1,1,3}$. This subdivision scheme is of particular interest because in this case a set of closed-form formulas for the resulting nonlinear subdivision operator is available and, consequently, the associated nonlinear pyramid transform can be implemented without resorting to relatively computationally expensive numerical algorithms [16], [4].

Main Result (Proposition 4.1). In this paper we prove a conjecture made in [16], [4], which states that the critical Hölder regularity of the above-mentioned nonlinear subdivision scheme $S$, denoted by $s_{\infty}(S)$, equals 1 , the same as that of an approximating linear subdivision scheme based on interpolation-imputation of midpoint values (denoted by $s_{\infty}(\bar{S})$ below.

Being a result of critical regularity, it improves earlier suboptimal results $s_{\infty}(S) \geq$ $\log _{3}\left(\frac{135}{121}\right)[16]$ and $s_{\infty}(S) \geq \log _{3}\left(\frac{135}{53}\right)[12]$.

The proof of our main result, while elementary, is not short and is subtle at places. Below we give an informal discussion of the main ideas and difficulties involved.

\subsection{Previous Work and Major Difficulties}

Below $S$ refers specifically to the triadic subdivision operator based on interpolation of median values by quadratics, and $\bar{S}$ refers to the corresponding linearization (denoted by $S_{Q_{L}}$ in Section 4$)$.

There is a local $[\mathbf{L}]$ way and a global $[\mathbf{G}]$ way to attack the sought-for Main Result (Proposition 4.1), each suffers from certain subtle difficulties.

\section{[L] Notice that, for an interval $I$,}

$$
\operatorname{median}(f \mid I)=f(\text { midpoint of } I), \quad \text { if } f \text { is monotone and continuous on } I \text {. }
$$

As the subdivision process $S^{j} v, j=1,2, \ldots$, smooths out the initial coarse data $v$, and smooth data are typically locally monotone-except at specific "chirping points" (see Figure 1), by (1.6) and the fact that $S$ acts locally and also that the Hölder regularity is a local concept, one expects that the Hölder smoothness of $S$ should be at least very close to that of a linear subdivision operator $\bar{S}$ based on interpolation-imputation of midpoint values.

The above intuition, unfortunately, cannot be easily turned into a proof for our Main Result (Proposition 4.1). In Figure 1, the successive blowups around $x=\frac{1}{2}$ show that $S^{\infty} \delta$ is not locally monotone at $\frac{1}{2}$. In a sense, such fractal behavior is not due to the nonlinearity presented in $S$-the fundamental limit function of the linear $\bar{S}$ shares the exact same fractal behavior. Nevertheless, the existence of such a chirping point means that it is not the case that the nonlinear subdivision process $S^{j} v, j=1,2, \ldots$, will always become 

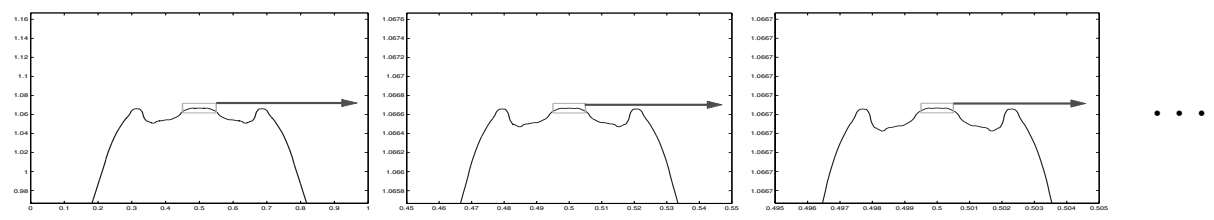

Fig. 1. Existence of a chirping point in $\varphi=S^{\infty} \delta$, the subdivision limit of the Kronecker delta sequence.

locally linear for large enough $j$; this accounts for some of the technical complication in the proof of our main result. A "fortunate" fact here is that the local Hölder regularity of $\varphi$ at $\frac{1}{2}$ is higher than the global regularity, so that the genuine nonlinear behavior of $S$ near $\frac{1}{2}$ does not affect our main result concerning global regularity. Ultimately, the difficulty is more serious than just the existence of a chirping point, see the concept of "bad zone" and its associated issues in Section 1.2 below.

[G] Standard linear subdivision calculations (more details below) show

$$
\begin{aligned}
\left\|\Delta \bar{S}^{j} v\right\|_{\ell^{\infty}} & \asymp j 3^{-j}, & & j \rightarrow \infty, \\
\left\|\Delta^{2} \bar{S}^{j} v\right\|_{\ell^{\infty}} & \asymp 3^{-j}, & & j \rightarrow \infty, \\
\left\|\Delta^{3} \bar{S}^{j} v\right\|_{\ell^{\infty}} & \asymp 3^{-j}, & & j \rightarrow \infty,
\end{aligned}
$$

for generic data $v$, e.g., $v=\delta$. In fact, any one of the above three statements can be used to conclude the fact that $s_{\infty}(\bar{S})=1$.

By certain approximation theoretic arguments (Section 3), the hoped for result $s_{\infty}(S)=1$ can be established if one can prove either (1.7) or (1.8) with $\bar{S}$ replaced by $S$. For ease of discussion in this section, we assume without proof that $s_{\infty}(S)=1$ can also be established if one manages to prove (1.9) with $\bar{S}$ replaced by $S$.

For $T: \ell^{\infty} \rightarrow \ell^{\infty}$, define its $r$ th-order shrinking factor by

$$
\varsigma(T, r):=\sup _{\substack{\Delta^{r} v \neq 0 \\ v \in \ell^{\infty}}} \frac{\left\|\Delta^{r} T v\right\|_{\ell^{\infty}}}{\left\|\Delta^{r} v\right\|_{\ell^{\infty}}} .
$$

Then we have the—in general suboptimal—bounds

$$
\begin{aligned}
\left\|\Delta^{r} \bar{S}^{j} v\right\|_{\ell^{\infty}}=O\left(\varsigma(\bar{S}, r)^{j}\right), & j \rightarrow \infty, \\
\left\|\Delta^{r} S^{j} v\right\|_{\ell^{\infty}}=O\left(\varsigma(S, r)^{j}\right), & j \rightarrow \infty .
\end{aligned}
$$

Various shrinking factors for $\bar{S}$ and $S$ are recorded in Table 1.

Since $\bar{S}$ is linear and $\Pi_{2}$ reproducing, there exist linear subdivision operators $\bar{S}^{[r]}$, $r=1,2,3$, such that $\Delta^{r} \circ \bar{S}=\bar{S}^{[r]} \circ \Delta^{r}, r=1,2,3$. By (1.14) there exists a homogeneous operator $S^{[1]}$ that satisfies $\Delta \circ S=S^{[1]} \circ \Delta$. Then it follows almost directly from definition that $\zeta(\bar{S}, r)=\left\|\bar{S}^{[r]}\right\|_{\ell^{\infty}}$, where $\left\|\bar{S}^{[r]}\right\|_{\ell^{\infty}}$ is the operator norm of $\bar{S}^{[r]}: \ell^{\infty} \rightarrow \ell^{\infty}$. 
Table 1. If we view $S$ as an nonlinear perturbation of $\bar{S}$, then nonlinearity "deteriorates" the degree of shrinkingness: $\varsigma(S, r)>\varsigma(\bar{S}, r)$ for each $r$.

\begin{tabular}{cccc}
\hline & $r=1$ & $r=2$ & $r=3$ \\
\hline$\varsigma(\bar{S}, r)$ & $=\left\|\bar{S}^{[1]}\right\|_{\ell^{\infty}}=\frac{7}{9}>\frac{1}{3}=\rho\left(\bar{S}^{[1]}\right)$ & $=\left\|\bar{S}^{[2]}\right\|_{\ell^{\infty}}=\frac{1}{3}=\rho\left(\bar{S}^{[2]}\right)$ & $=\left\|\bar{S}^{[3]}\right\|_{\ell^{\infty}}=\frac{1}{3}=\rho\left(\bar{S}^{[3]}\right)$ \\
$\varsigma(S, r)$ & $=\varsigma\left(S^{[1]}, 0\right)=\frac{121}{135}[16]$ & $\frac{53}{135}[12]$ & $+\infty$ \\
\hline
\end{tabular}

For a linear operator $T: \ell^{\infty} \rightarrow \ell^{\infty}$, the standard spectral radius formula reads

$$
\rho(T)=\lim _{j \rightarrow \infty}\left\|T^{j}\right\|^{1 / j}=\inf _{j \geq 0}\left\|T^{j}\right\|^{1 / j} .
$$

(Here and below $\|T\|$ always refers to the operator norm of $T$ as an operator on $\ell^{\infty}$.) The critical Hölder regularity of $\bar{S}$ is determined by the exact asymptotic behavior of the left-hand sides of (1.7)-(1.9), which, in turn, can be determined from the spectral radii $\rho\left(\bar{S}^{[r]}\right), r=1,2,3$. Calculating $\|T\|$ is straightforward, while calculating the spectral quantity $\rho(T)$ is usually difficult, as typically not only is it the case that $\rho(T)<\|T\|$ but also that

$$
\rho(T)<\left\|T^{j}\right\|^{1 / j} \quad \text { for all } j
$$

For instance, (1.12) holds if $T=\bar{S}^{[1]}$; but it happens that

$$
\rho\left(\bar{S}^{[r]}\right)=\left\|\bar{S}^{[r]}\right\|=\frac{1}{3}, \quad r=2,3,
$$

and (1.8)-(1.9) follow from the above. The proof of (1.13) requires the help of a positivity argument in Fourier domain, so it relies heavily on the linearity structure.

Based on the "fortunate fact" (1.13) and our intuition that $S \approx \bar{S}$, one may expect that the bound (1.11) should be rather suboptimal in the case when $r=1$ but nearly optimal when $r=2$ and $r=3$, this is because the asymptotic bound on the right-hand side of (1.10) is indeed, by (1.13), the best possible for either $r=2$ or $r=3$. It turns out that this speculation is only true when $r=2$ and, as it stands, is completely wrong when $r=3$. The nonlinear subdivision operator $S$, while based on a local nonlinear quadratic interpolation process, does not reproduce quadratic data, i.e., there exists $v$ such that $\Delta^{3} v=0$ but $\Delta^{3} S v \neq 0$, in other words,

$$
\varsigma(S, 3)=+\infty
$$

On the other hand, $S$ reproduces linear data (see Section 2.1) and $\varsigma(S, 2)$ is finite, Oswald's calculation [12] gives

$$
\varsigma(S, 2)=\frac{53}{121}>\frac{1}{3}
$$

which, as expected, yields the decent but still suboptimal bound $\left\|\Delta^{2} S^{j} v\right\|_{\ell^{\infty}}=O\left(\left(\frac{35}{121}\right)^{j}\right)$.

The difficulty we are facing is that the proof of either (1.8) or (1.9) with $\bar{S}$ replaced by $S$ cannot be based directly on calculating $\varsigma(S, r), r=2,3$. The fact that $\varsigma(S, r)>\varsigma(\bar{S}, r)$ is a genuine bad effect of nonlinearity. 


\subsection{Overview of the Proof}

The first observation is that any median-interpolating subdivision operator $S: \ell(\mathbb{Z}) \rightarrow$ $\ell(\mathbb{Z})$ (see [4], [13] or Section 5), while nonlinear, is offset invariant (Definition 2.3) as well as homogeneous, i.e.,

$$
S\left(c v+c^{\prime}\right)=c S(v)+c^{\prime}, \quad \forall c, c^{\prime} \in \mathbb{R}, \quad v \in \ell(\mathbb{Z})
$$

this follows from the following affine-invariant property of median:

$$
\operatorname{median}\left(c f+c^{\prime} \mid I\right)=c \cdot \operatorname{median}(f \mid I)+c^{\prime}, \quad \forall c, c^{\prime} \in \mathbb{R}, \quad f \in C(I) .
$$

By (1.14) there exists a homogeneous operator $S^{[1]}$ that satisfies $\Delta \circ S=S^{[1]} \circ \Delta$. However, as $S^{[1]}$ is not offset invariant, there does not exist a local operator $S^{[2]}$ that satisfies $\Delta^{2} \circ S=S^{[2]} \circ \Delta^{2}$. We observe that $S^{[1]}$, being a local map, can be represented by three nonlinear maps $P^{l}: \mathbb{R}^{3} \rightarrow \mathbb{R}^{3}, l=1,2,3$, in such a way that, for any length 3 segment $w_{j}$ of the sequence $\left(S^{[1]}\right)^{j} v$, there exist a length 3 segment $w_{0}$ of $v$ and a sequence of digits $\varepsilon_{i} \in\{1,2,3\}, i=1, \ldots, j$, such that

$$
P^{\varepsilon_{j}} \circ \cdots \circ P^{\varepsilon_{1}}\left(w_{0}\right)=w_{j}
$$

A specific local calculation verifies that $\sup _{v \in \ell^{\infty}}\left\|\Delta^{2} S^{j} v\right\|_{\ell^{\infty}}=\Omega\left(\left(\frac{1}{3}\right)^{j}\right)$, so to prove (1.8) with $\bar{S}$ replaced by $S$ one needs to show $\left\|\Delta^{2} S^{j} v\right\|_{\ell^{\infty}}=O\left(\left(\frac{1}{3}\right)^{j}\right)$ or, equivalently,

$$
\left\|\Delta\left(S^{[1]}\right)^{j} v\right\|_{\ell^{\infty}}=O\left(\left(\frac{1}{3}\right)^{j}\right), \quad \forall \text { finitely supported } v .
$$

The nonexistence of $S^{[2]}$ means one cannot determine $\Delta P^{l}(w)$ from $\Delta w$. However, it is not hard to see that $\Delta P^{l}(w)$ can be determined from $\Delta w$ and $[w]_{\sim}$, here $[w]_{\sim}$ is an element in the real projective space $\mathbb{P}\left(\mathbb{R}^{3}\right)$ defined by $(2.15)-(2.16)$, i.e., there exist maps $F^{l}: \mathbb{R}^{2} \times \mathbb{P}\left(\mathbb{R}^{3}\right) \rightarrow \mathbb{R}^{2}$ such that

$$
F^{l}\left(\Delta w,[w]_{\sim}\right)=\Delta P^{l}(w) .
$$

On the other hand, as $S^{[1]}$ is homogeneous, so are $P^{l}, l=1,2,3$, hence (Lemma 2.8) there exist quotient maps $\varrho_{l}: \mathbb{P}\left(\mathbb{R}^{3}\right) \rightarrow \mathbb{P}\left(\mathbb{R}^{3}\right)$ such that

$$
\varrho_{l}\left([w]_{\sim}\right)=\left[P^{l}(w)\right]_{\sim} .
$$

While Oswald's result shows that

$$
\varsigma(S, 2)=\varsigma\left(S^{[1]}, 1\right)=\sup _{[w]_{\sim} \in \mathbb{P}\left(\mathbb{R}^{3}\right)} \max _{l=1,2,3} \frac{\left\|F^{l}\left(\Delta w,[w]_{\sim}\right)\right\|_{\infty}}{\|\Delta w\|_{\infty}}=\frac{35}{121}>\frac{1}{3},
$$

we observe (in Figure 3 ) that indeed for most points $[w]_{\sim}$ in the projective plane $\mathbb{P}\left(\mathbb{R}^{3}\right)$ we have

$$
\varsigma\left([w]_{\sim}\right):=\max _{l=1,2,3} \frac{\left\|F^{l}\left(\Delta w,[w]_{\sim}\right)\right\|_{\infty}}{\|\Delta w\|_{\infty}} \leq \frac{1}{3}
$$

in other words, only for points $[w] \sim$ in a small "bad zone" $\mathcal{B} \subset \mathbb{P}\left(\mathbb{R}^{3}\right)$ are (1.20) violated. 
Now, to show (1.17) it suffices, by virtue of (1.16) and (1.19), to show that there exists a universal constant $C>0$ such that for any $\theta \in \mathbb{P}\left(\mathbb{R}^{3}\right)$ and for any sequence $\left(\varepsilon_{i}\right)_{i=1}^{\infty}$, $\varepsilon_{i} \in\{1,2,3\}$, if we define $\theta_{0}:=\theta$,

$$
\theta_{j}:=\varrho_{\varepsilon_{j}}\left(\theta_{j-1}\right), \quad j=1,2, \ldots,
$$

then

$$
\varsigma\left(\theta_{j-1}\right) \cdots \varsigma\left(\theta_{0}\right) \leq C\left(\frac{1}{3}\right)^{j}, \quad \forall j
$$

So eventually the problem boils down to understanding the distribution of the function $\varsigma: \mathbb{P}\left(\mathbb{R}^{3}\right) \rightarrow[0, \infty)$ and the joint dynamics of the three maps $\varrho_{l}$.

A Compensation Argument. Through oral communications with researchers who had encountered nonlinear subdivision schemes (notably Nira Dyn working on subdivision of Lie group valued data and Ingrid Daubechies working on normal multiresolution [3]), we had heard more than once the comment that when a nonlinear subdivision scheme shares the same smoothness with an approximating linear subdivision scheme, it is likely because nonlinearity "goes away" in the limit of the subdivision process. Extrapolating from this comment, one may be led to speculate that (1.22) is true because

(*) there is a finite integer $m>0$ such that for any sequence $\left(\varepsilon_{i}\right)_{i=1}^{\infty}, \varepsilon_{i} \in\{1,2,3\}$, $\theta_{j} \in \mathcal{B}$ for at most $m$ different $j$ 's.

No doubt $(*)$ implies (1.22), but at this point we do not know if $(*)$ is true: we cannot rule out the lurking possibility that for a carefully chosen $\left(\varepsilon_{i}\right)_{i},\left(\theta_{j}\right)_{j=0}^{\infty}$ may go in and out of the bad zone arbitrarily often or even infinitely often. We do not know if the "bad effect" of nonlinearity always "goes away" after a number of subdivision steps.

Instead, our proof of (1.22) is based on a "compensation argument": whenever more than two "bad steps" occur, i.e., $\varsigma\left(\theta_{j}\right)>\frac{1}{3}$ for more than two different $j$ 's, we show that there always exist some "good steps," i.e., $j^{\prime}$ such that $\varsigma\left(\theta_{j^{\prime}}\right)<\frac{1}{3}$ is small enough to compensate for the effect of the excessive bad steps, so overall (1.22) holds for a constant $C$ independent of $\left(\varepsilon_{i}\right)_{i}$ and of $\theta_{0}$. To this end, the closed-form formulas possessed by $S$ are used quite extensively.

\subsection{Contents}

Section 2 collects all the relevant basic facts for general homogeneous and affine invariant subdivision operators, then we establish, in a general context, the key commutation relations (1.18)-(1.19). Section 3 presents the basic approximation theoretical arguments which eventually, in Theorem 3.4, reduce a general smoothness problem to an estimate of the form (1.22). Section 4 lays out the main argument, with the compensation argument mentioned above as the key step, in order to establish the bound (1.22), thus proving the Main Result (Proposition 4.1). Some of the calculations involved in the proof are postponed to the Appendix. In Section 5 we derive, using linerization heuristics, a generalized conjecture (of which Proposition 4.1 is a very special case) speculating that the smoothness of a $p$-mean subdivision scheme is the same as that of an approximating 
linear subdivision scheme. A closed-form formula for the mask of the latter linear subdivision scheme is derived, and numerical experiments are given to justify the conjecture as well.

\section{Homogeneous and Affine Invariant Subdivision Operators}

Definition 2.1. A (not necessarily linear) operator $S: \ell(\mathbb{Z}) \rightarrow \ell(\mathbb{Z})$ is local if there exists a constant integer $D \geq 0$ such that each $(S m)_{k}$ depends only on $D$ entries of $m$. For a local operator, the minimum $D$ that satisfies the definition is called the locality factor of $S$.

A local operator $S: \ell(\mathbb{Z}) \rightarrow \ell(\mathbb{Z})$ is called a subdivision operator if it satisfies

$$
S(m \cdot-k)=(S m)_{--d k} .
$$

Here $d \geq 2$ is an integer and is referred to as the dilation factor of the subdivision operator $S$.

In this paper, a shift-invariant operator refers to a local operator $T: \ell(\mathbb{Z}) \rightarrow \ell(\mathbb{Z})$ which satisfies

$$
T(m \cdot-k)=(T m)_{--k} .
$$

Notice that if $S$ is a subdivision operator and $T$ is a shift-invariant operator, then $S \circ T$ and $T \circ S$ are subdivision operators.

One can equivalently redefine a subdivision operator as follows. Let $Q=\left(Q_{1}, \ldots, Q_{d}\right)$ : $\mathbb{R}^{D} \rightarrow \mathbb{R}^{d}$ where $d \geq 2$. Let $n \in \mathbb{Z}$. The subdivision operator $S:=S_{Q, n}: \ell(\mathbb{Z}) \rightarrow \ell(\mathbb{Z})$ associated with $Q$ and (phase factor) $n$ is defined by

$$
(S m)_{d i+l-1}=Q_{l}\left(m_{i-n+1}, \ldots, m_{i-n+D}\right), \quad l=1, \ldots, d, \quad i \in \mathbb{Z} .
$$

Clearly $S_{Q, n}$ is a subdivision operator and, in particular, is local. We assume by convention that $D$, the dimension of the domain of the map $Q$, is the same as the locality factor of $S_{Q, n}$.

When $D=0,(2.3)$ is interpreted as $(S m)_{d i+l-1}=Q_{l}$ for some constants $Q_{l}, l=$ $1, \ldots, d$. However, unless otherwise stated, we assume that any subdivision operator is nonconstant and hence has a locality factor $D \geq 1$.

When $Q: \mathbb{R}^{D} \rightarrow \mathbb{R}^{d}$ is a linear map, then it can be represented by a $d \times D$ matrix $\left(q_{i, j}\right)_{1 \leq i \leq d, 1 \leq j \leq D}$ and $S_{Q, n}$ is a standard linear subdivision operator

$$
(S x)_{k}=\sum_{i \in \mathbb{Z}} a_{k-d i} x_{i}
$$

with mask $\left(a_{i}\right)$ uniquely determined by $Q$ and $n$.

For $x \in \ell(\mathbb{Z})$ or $\mathbb{R}^{N}$, we introduce

$$
x_{k}^{[p]}:=\left(x_{k}, \ldots, x_{k+p-1}\right) \in \mathbb{R}^{p},
$$

( $k$ has to satisfy $k+p-1 \leq N$ if $x \in \mathbb{R}^{N}$ ) as shorthand to denote a length $p$ segment 
of $x$. Under this notation, we can rewrite (2.3) as

$$
(S m)_{d i}^{[d]}=Q\left(m_{i-n+1}^{[D]}\right), \quad \forall i \in \mathbb{Z} .
$$

Since a subdivision operator $S$ acts locally, one can determine a length $(R-D+1) d$ segment of $S(m)$ from a length $R(\geq D)$ segment of a sequence $m$. In view of that, when $x \in \mathbb{R}^{R}$ for $R \geq D$, we write

$$
S(x)=\left(Q_{1}\left(x_{1}^{[D]}\right), \ldots, Q_{d}\left(x_{1}^{[D]}\right), \ldots, Q_{1}\left(x_{R-D+1}^{[D]}\right), \ldots, Q_{d}\left(x_{R-D+1}^{[D]}\right)\right) \in \mathbb{R}^{(R-D+1) d} .
$$

Thus $S(x)$ is "all the data that $S$ can generate by knowing only the segment $x$ of a sequence." It should be easy to distinguish from the context whether we refer to $S$ as a map from $\mathbb{R}^{R}$ to $\mathbb{R}^{(R-D+1) d}$ or as a map from $\ell(\mathbb{Z})$ to itself.

Notice also that if $(R-D+1) d \geq R+d-1$, i.e.,

$$
R \geq \frac{D d-1}{d-1} \quad(\geq D)
$$

then $(S m)_{d i+l-1}^{[R]}, l=1, \ldots, d$, can be determined from $m_{i-n+1}^{[R]}$; in particular, every length $R$ segment of $S(m)$ can be determined from a certain length $R$ segment of $m$. Due to $(2.1)$ we can also make the following finer statement:

Proposition 2.2. Given a subdivision operator $S_{Q, n}$, for any large enough $R$, there exist $P^{1}, \ldots, P^{d}: \mathbb{R}^{R} \rightarrow \mathbb{R}^{R}$ such that, for any sequence $m$,

$$
(S m)_{d i+l-1}^{[R]}=P^{l}\left(m_{i-n+1}^{[R]}\right), \quad \forall i \in \mathbb{Z}, \quad l=1, \ldots, d .
$$

Proof. Let $R$ be an integer that satisfies (2.5). For $l=1, \ldots, d$, define $P^{l}: \mathbb{R}^{R} \rightarrow \mathbb{R}^{R}$ by $P^{l}(x)=(S x)_{l}^{[R]}$, then (2.6) holds according to $(2.3)$.

In general, $\lceil(D d-1) /(d-1)\rceil$ is not the smallest $R$ that allows for the existence of maps $P^{l}: \mathbb{R}^{R} \rightarrow \mathbb{R}^{R}$ which satisfy (2.6). Hence we define

$$
R_{S}:=\min \{R: R \text { has the property in Proposition } 2.2\}
$$

to be the neighborhood factor of the subdivision operator $S$. As an example, consider a linear subdivision operator $S$ with a mask of support length 3 and dilator factor $d=2$, then $D=2$ and $\lceil(D d-1) /(d-1)\rceil=3$, but it can be observed that $R_{S}=2$. Since any length $R_{S}$ segment of $m$ determines a segment of $S m$ of length at least $d$, and that the locality factor $D$ of $S$ is the smallest integer with this property, so

$$
R_{S} \geq D
$$

If $X$ and $Y$ are vector spaces over $\mathbb{R}$, recall that a map $M: X \rightarrow Y$ is homogeneous if

$$
M(c x)=c M(x), \quad \forall x \in X, \quad c \in \mathbb{R} .
$$

Definition 2.3. Let $M: V \rightarrow W$, where $V$ and $W$ are any one of $\ell(\mathbb{Z})$ or $\mathbb{R}^{n}, n=$ $1,2, \ldots$

(i) $M$ is offset invariant if $M(m+c)=M(m)+c$ for any $m \in V$ and $c \in \mathbb{R}$. Here, if $v$ is an element in $\ell(\mathbb{Z})$ or $\mathbb{R}^{n}$ and $c \in \mathbb{R}$, then $v+c$ refers to the element 
in the same space defined by

$$
(v+c)_{i}=v_{i}+c, \quad \forall i .
$$

(ii) $M$ is affine invariant if $M$ is both offset invariant and homogeneous.

The following is obvious from (2.3).

Proposition 2.4. Let $S=S_{Q, n}$ be a subdivision operator.

(i) $S$ is offset invariant $\Leftrightarrow Q$ is offset invariant.

(ii) $S$ is homogeneous $\Leftrightarrow Q$ is homogeneous.

(iii) $S$ is affine invariant $\Leftrightarrow Q$ is affine invariant.

\subsection{Commutation Relations}

Let $\Delta: \ell(\mathbb{Z}) \rightarrow \ell(\mathbb{Z})$ or $\mathbb{R}^{N} \rightarrow \mathbb{R}^{N-1}(N \geq 2)$ be the forward differencing operator, defined by $(\Delta m)_{i}=m_{i+1}-m_{i}$. Let

$$
\Delta^{1}:=\Delta, \quad \Delta^{r+1}:=\Delta \circ \Delta^{r} .
$$

(Clearly we are committing an abuse of notation when $\Delta$ acts on finite vectors, in this case $\Delta^{r}$ maps from $\mathbb{R}^{N}$ to $\mathbb{R}^{N-r}, N \geq r+1$.)

If a subdivision operator $S$ is offset invariant, then the difference sequences $\Delta\left(m^{j}\right), j=$ $0,1, \ldots$, where $m^{0} \in \ell(\mathbb{Z}), m^{j+1}=S m^{j}$, follow another subdivision scheme. This observation is used already in the earlier works [16], [4], [12], [11].

Theorem 2.5. If a subdivision operator $S$ is offset invariant, then there exists an unique subdivision operator $S^{[1]}$ with the same dilation factor as $S$ such that

$$
S^{[1]} \circ \Delta=\Delta \circ S .
$$

Proof. The uniqueness basically follows from the surjectivity of $\Delta$ as an operator on $\ell(\mathbb{Z})$. Define $\Sigma: \ell(\mathbb{Z}) \rightarrow \ell(\mathbb{Z})$ by

$$
(\Sigma y)_{k}= \begin{cases}\sum_{i=0}^{k} y_{i}, & \text { if } k \geq 0 \\ -\sum_{i=k}^{0} y_{i}, & \text { if } k<0 .\end{cases}
$$

We have $\Delta \circ \Sigma=$ id. If $S^{[1]}$ is any operator on $\ell(\mathbb{Z})$ that satisfies (2.8),

$$
S^{[1]} y=S^{[1]} \Delta \Sigma y=\Delta S \Sigma y \text {. }
$$

Thus $S^{[1]}=\Delta \circ S \circ \Sigma$ is the only possibility.

While it is clear that $\Delta \circ S \circ \Sigma$ satisfies (2.1), it may be less clear whether the operator is local, as $\Sigma$ is not local. The locality property of $\Delta \circ S \circ \Sigma$ hinges on the assumption that $S$ is offset invariant. We prove locality by establishing a different representation of $S^{[1]}$.

It follows from Proposition 2.4(i) and (2.1) that $\forall m \in \ell(\mathbb{Z}), \forall i \in \mathbb{Z}, l=1, \ldots, d-1$, 
we have

$$
\begin{aligned}
(\Delta(S m))_{d i+l-1}= & (S m)_{d i+l}-(S m)_{d i+l-1} \\
= & Q_{l+1}\left(m_{i-n+1}^{[D]}\right)-Q_{l}\left(m_{i-n+1}^{[D]}\right) \\
= & {\left[m_{i-n+1}+Q_{l+1}\left(0, m_{i-n+2}-m_{i-n+1}, \ldots, m_{i-n+D}-m_{i-n+1}\right)\right] } \\
& -\left[m_{i-n+1}+Q_{l}\left(0, m_{i-n+2}-m_{i-n+1}, \ldots, m_{i-n+D}-m_{i-n+1}\right)\right] \\
= & \left(Q_{l+1}-Q_{l}\right)\left(0,(\Delta m)_{i-n+1}, \ldots, \sum_{j=1}^{D-1}(\Delta m)_{i-n+j}\right)
\end{aligned}
$$

and

$$
\begin{aligned}
(\Delta(S m))_{d i+d-1}= & (S m)_{d i+d}-(S m)_{d i+d-1} \\
= & Q_{1}\left(m_{i-n+2}^{[D]}\right)-Q_{d}\left(m_{i-n+1}^{[D]}\right) \\
= & {\left[m_{i-n+1}+Q_{1}\left(m_{i-n+2}-m_{i-n+1}, \ldots, m_{i-n+D+1}-m_{i-n+1}\right)\right] } \\
& -\left[m_{i-n+1}+Q_{d}\left(0, m_{i-n+2}-m_{i-n+1}, \ldots, m_{i-n+D}-m_{i-n+1}\right)\right] \\
= & Q_{1}\left((\Delta m)_{i-n+1}, \ldots, \sum_{j=1}^{D}(\Delta m)_{i-n+j}\right) \\
& -Q_{d}\left(0,(\Delta m)_{i-n+1}, \ldots, \sum_{j=1}^{D-1}(\Delta m)_{i-n+j}\right)
\end{aligned}
$$

Let $Q^{1}: \mathbb{R}^{D} \rightarrow \mathbb{R}^{d}$ be defined by

$$
Q_{l}^{1}\left(x_{1}, \ldots, x_{D}\right)= \begin{cases}\left(Q_{l+1}-Q_{l}\right)\left(0, x_{1}, \ldots, \sum_{k=1}^{D-1} x_{k}\right), & \text { if } 1 \leq l \leq d-1, \\ Q_{1}\left(x_{1}, \ldots, \sum_{k=1}^{D} x_{k}\right)-Q_{d}\left(0, x_{1}, \ldots, \sum_{k=1}^{D-1} x_{k}\right), & \text { if } l=d .\end{cases}
$$

Then it follows from (2.9) and (2.10) that $\forall m \in l(\mathbb{Z}), \forall i \in \mathbb{Z}, l=1, \ldots, d$,

$$
(\Delta(S m))_{d i+l-1}=Q_{l}^{1}\left((\Delta m)_{i-n+1}^{[D]}\right) .
$$

Let $S^{[1]}:=S_{Q^{1}, n}$, then it follows from (2.12) that (2.8) holds.

The following is a corollary (of the proof) of Theorem 2.5.

Corollary 2.6. If $S$ is affine invariant, then $S^{[1]}$ (exists and) is homogeneous.

Proof. Since $S$ is homogeneous, $Q$ is homogeneous. It follows from (2.11) and (2.12) that $Q^{1}$ is also homogeneous. Therefore $S^{[1]}$ is homogeneous by Proposition 2.4(ii).

Proposition 2.7. If a subdivision operator $S$ is offset invariant, then $R_{S^{[1]}} \leq R_{S}-1$. 
Proof. Let $P^{1}, \ldots, P^{d}: \mathbb{R}^{R} \rightarrow \mathbb{R}^{R}$ be maps that satisfy (2.6) with $R=R_{S}$. Since $S$ is offset invariant, so is $Q$ and it is evident from (2.6) that any $P^{l}$ is also offset invariant. Let $\left(S^{[1]}(\Delta m)\right)_{d i+l-1}^{[R-1]}$ be a length $R-1$ segment of $S^{[1]}(\Delta m)$, where $m \in \ell(\mathbb{Z}), i \in$ $\mathbb{Z}, 1 \leq l \leq d$, and $d$ is the dilation factor of $S$. Then, for $1 \leq k \leq R-1$,

$$
\begin{aligned}
\left(S^{[1]}(\Delta m)\right)_{d i+l+k-2}= & (\Delta(S m))_{d i+l+k-2} \\
= & (S m)_{d i+l+k-1}-(S m)_{d i+l+k-2} \\
= & P_{k+1}^{l}\left(m_{i-n+1}^{[R]}\right)-P_{k}^{l}\left(m_{i-n+1}^{[R]}\right) \\
= & {\left[m_{i-n+1}+P_{k+1}^{l}\left(0, m_{i-n+2}-m_{i-n+1}, \ldots, m_{i-n+R}-m_{i-n+1}\right)\right] } \\
& -\left[m_{i-n+1}+P_{k}^{l}\left(0, m_{i-n+2}-m_{i-n+1}, \ldots, m_{i-n+R}-m_{i-n+1}\right)\right] \\
= & \left(P_{k+1}^{l}-P_{k}^{l}\right)\left(0,(\Delta m)_{i-n+1}, \ldots, \sum_{j=1}^{R-1}(\Delta m)_{i-n+j}\right) .
\end{aligned}
$$

Let $\bar{P}^{l}: \mathbb{R}^{R-1} \rightarrow \mathbb{R}^{R-1}$ be defined by

$$
\bar{P}_{k}^{l}\left(x_{1}, \ldots, x_{R-1}\right)=\left(P_{k+1}^{l}-P_{k}^{l}\right)\left(0, x_{1}, \ldots, \sum_{i=1}^{R-1} x_{i}\right), \quad k=1, \ldots, R-1
$$

Then

$$
\left(S^{[1]}(\Delta m)\right)_{d i+l-1}^{[R-1]}=\bar{P}^{l}\left((\Delta m)_{i-n+1}^{[R-1]}\right) .
$$

Therefore, $R_{S^{[1]}} \leq R-1=R_{S}-1$.

Let $X$ be any vector space. Define the quotient space

$$
\mathbb{P}(X):=X / \sim:=\left\{[x]_{\sim}: x \in X\right\}
$$

where $\sim$ is the equivalent relation defined by

$$
x \sim y \Leftrightarrow \exists c \neq 0 \quad \text { such that } \quad x=c y .
$$

$\left(\mathbb{P}\left(\mathbb{R}^{n}\right)\right.$ with the point $[\mathbf{0}] \sim$ removed possesses a natural differentiable structure and is usually denoted by $\mathbb{P}^{n-1}$ in differential geometry.)

Given $\theta \in \mathbb{P}(\ell(\mathbb{Z}))$, one can define:

a shift:

$$
\theta \cdot-k \in \mathbb{P}(\ell(\mathbb{Z})),
$$

a segment:

$$
\theta_{i}^{[N]} \in \mathbb{P}\left(\mathbb{R}^{N}\right),
$$

or the $k$ th-order difference:

$$
\Delta^{k} \theta \in \mathbb{P}(\ell(\mathbb{Z}))
$$

of $\theta$ in the obvious way. In fact, all these can be viewed as special applications of the following well-known principle: 
Lemma 2.8. Let $X$ and $Y$ be vector spaces over a common field. If $f: X \rightarrow Y$ is homogeneous, then $f$ induces a map $[f]: \mathbb{P}(X) \rightarrow \mathbb{P}(Y)$ via the formula

$$
[f]\left([x]_{\sim}\right):=[f(x)]_{\sim} .
$$

Proof. We need to prove that $[f]$ is well defined. Let $y, z \in[x] \sim$. Then $\exists c \in \mathbb{R}, c \neq 0$, such that $y=c z$. Since $f$ is homogeneous, $f(y)=c f(z)$. So $[f(y)]_{\sim}=[f(z)]_{\sim}$, and $[f]$ is well defined.

If $T: \ell(\mathbb{Z}) \rightarrow \ell(\mathbb{Z})$ is a homogeneous subdivision operator, then it is quite clear that $[T]$ inherits the subdivision properties from $T$. We have

$$
[T](\vartheta \cdot-k)=([T](\vartheta))_{--d k} .
$$

Also, any segment of $[T](\vartheta)$ of the form $([T](\vartheta))_{d i}^{[d]}$ can be determined from a length $D$ segment of $\vartheta$; and any length $R$ segment of $[T](\vartheta), R \geq R_{T}$, can be determined from a length $R$ segment of $\vartheta$.

Definition 2.9. A subdivision operator $S$ reproduces $\Pi_{r}(r \geq-1)$ if

$$
\left.S\left(\left.\Pi_{r}\right|_{\mathbb{Z}}\right) \subset \Pi_{r}\right|_{\mathbb{Z}}
$$

i.e., for every polynomial $p \in \Pi_{r}, \exists q \in \Pi_{r}$ such that $S\left(\left.p\right|_{\mathbb{Z}}\right)=\left.q\right|_{\mathbb{Z}}$. A subdivision operator $S$ is of order $r+1$ if it reproduces $\Pi_{l}$ for all $l=0, \ldots, r$.

For instance, an offset invariant subdivision operator reproduces $\Pi_{0}$.

The following proposition is an easy consequence of the fact that if $r \geq 0$ and $x \in \mathbb{R}^{r+2}$, then $\Delta^{r+1} x=0$ if and only if $x_{i}=p(i), i=1, \ldots, r+2$, for some $p \in \Pi_{r}$.

Proposition 2.10. Let $S_{Q, n}$ be a subdivision operator. The following statements are equivalent:

(i) $S$ reproduces $\Pi_{r}$.

(ii) For any $m \in \ell(\mathbb{Z}), \Delta^{r+1} m=0 \Rightarrow \Delta^{r+1}(S m)=0$.

(iii) Let $R \geq \max (r+2, D+(r+2) / d-1)$ be given. (So if $x \in \mathbb{R}^{R}$, the lengths of $x$ and $S x$ are at least $r+2$.) For any $x \in \mathbb{R}^{R}, \Delta^{r+1} x=0 \Rightarrow \Delta^{r+1}(S x)=0$.

Our next result complements the following well-known observation in the theory of linear subdivision schemes: When $S$ is a linear subdivision operator which also reproduces $\Pi_{r-1}$, there exists an unique (linear) subdivision operator $S^{[r]}$ such that

$$
\Delta^{r} \circ S=S^{[r]} \circ \Delta^{r} .
$$

While the same does not hold in the nonlinear case, we now prove that for a homogeneous subdivision operator $T$ which reproduces $\Pi_{r-1}$, every element of $\Delta^{r}(T x)$ can be expressed in terms of a local quasi-linear combination of elements in $\Delta^{r} x$, with coefficients dependent only locally on $[x] \sim$. 
Theorem 2.11. Let $S=S_{Q, n}$ be a homogeneous subdivision operator that reproduces $\Pi_{r-1}$ for some $r \geq 0$. Let $d$ and $R_{S}$ be the dilation and neighborhood factors of $S$, respectively. Let

$$
R \geq \max \left(R_{S}, r+1\right) .
$$

Let $P^{l}: \mathbb{R}^{R} \rightarrow \mathbb{R}^{R}$ be the maps defined in Proposition 2.2 which (redundantly) represent $S$ in such a way that

$$
(S x)_{d i+l-1}^{[R]}=P^{l}\left(x_{i-n+1}^{[R]}\right), \quad \forall i \in \mathbb{Z}, \quad l=1, \ldots, d .
$$

Then there exist maps

$$
F^{l}: \mathbb{R}^{R-r} \times \mathbb{P}\left(\mathbb{R}^{R}\right) \rightarrow \mathbb{R}^{R-r}, \quad l=1, \ldots, d,
$$

such that

$$
\Delta^{r}\left(P^{l}(x)\right)=F^{l}\left(\Delta^{r} x,[x]_{\sim}\right), \quad \forall x \in \mathbb{R}^{R}, \quad l=1, \ldots, d .
$$

Moreover, for any fixed $\theta, F^{l}(\cdot, \theta)$ is a linear map with rank $\leq 1$.

We shall prove this theorem by constructing the maps $F^{l}$ explicitly, to do so we first make the observation that a vector $x \in \mathbb{R}^{R}$ can be uniquely determined from $[x]_{\sim}$ and $\Delta^{r} x, r<R$, as long as $\Delta^{r} x \neq 0$; below we actually prove more than just this. (The complication is largely attributable to the simple fact that $[x]_{\sim}$ and $\Delta^{r} x$ can provide either very redundant or insufficient information for determining $x$.)

Lemma 2.12. Let $0 \leq r<R$. There exists a map $L: \mathbb{R}^{R-r} \times \mathbb{P}\left(\mathbb{R}^{R}\right) \rightarrow \mathbb{R}^{R}$ such that

$$
L\left(\Delta^{r} x,[x] \sim\right)=x, \quad \forall x \in \mathbb{R}^{R} \text { such that } \Delta^{r} x \neq 0 ;
$$

moreover, $L$ can be chosen to have the following quasi-linear form:

$$
L(y, \theta)=M(\theta) y, \quad M: \mathbb{P}\left(\mathbb{R}^{R}\right) \rightarrow \mathbb{R}^{R \times(R-r)} .
$$

Proof. Let $\Gamma$ be the subspace $\left\{x \in \mathbb{R}^{R}: \Delta^{r} x=0\right\}$. Let $\Sigma_{r}$ be any linear map such that $\Delta^{r} \Sigma_{r}$ is the identity map on $\mathbb{R}^{R-r}$.

If $\theta \subseteq \Gamma$, simply set $M(\theta)=0$, so $L(y, \theta)=0$ for all $y$; otherwise, $\theta=[z] \sim$ $(=\{c z: c \neq 0\})$ for some $z \notin \Gamma$ so that the line $\Theta:=\{c z: c \in \mathbb{R}\}$ and the plane $\Sigma_{r} y+\Gamma$ are not parallel. Consider

$$
\begin{aligned}
L(y, \theta) & :=\underset{w \in \Theta}{\arg \min } \operatorname{dist}\left(w, \Sigma_{r} y+\Gamma\right) \\
& :=\underset{w \in \Theta}{\arg \min } \min _{w^{\prime} \in \Sigma_{r} y+\Gamma}\left\|w-w^{\prime}\right\|_{2} .
\end{aligned}
$$

We need to show that in (2.23) a minimizer exists and is unique (so $L$ is well defined), and also that $L$ can be written in the form (2.22). First, notice that

$$
\min _{w \in \Theta, w^{\prime} \in \Sigma_{r} y+\Gamma}\left\|w-w^{\prime}\right\|_{2}=\min _{v \in \Theta+\Gamma}\left\|v-\Sigma_{r} y\right\|_{2} .
$$


Since $\Theta$ and $\Gamma$ are independent subspaces of $\mathbb{R}^{R}$, for every $x \in \Theta+\Gamma$ there exist unique $x_{\Theta} \in \Theta, x_{\Gamma} \in \Gamma$ such that $x=x_{\Theta}+x_{\Gamma}$; moreover, there exists a linear map $G(\theta): \Theta+\Gamma \rightarrow \Theta$ such that $x_{\Theta}=G(\theta) x$. Next, let $P_{\Theta+\Gamma}$ be the $\left(L^{2}\right.$-)projector of $\mathbb{R}^{R}$ onto $\Theta+\Gamma$. Then it is evident that

$$
G(\theta) P_{\Theta+\Gamma} \Sigma_{r} y
$$

uniquely solves the minimization problem on the right-hand side of (2.23).

To conclude, we define $L$ via $M$ by (2.22), and we define $M$ as

$$
M(\theta):= \begin{cases}0, & \theta \subseteq \Gamma, \\ G(\theta) P_{\Theta+\Gamma} \Sigma_{r}, & \text { otherwise. }\end{cases}
$$

Finally, if $\theta=[x]_{\sim}$ and $y=\Delta^{r} x \neq 0$, then $x \notin \Gamma$ and $\theta \nsubseteq \Gamma$. In this case $\Theta$ and $\Sigma_{r} y+\Gamma$ have an unique intersecting point, namely, $x$; in other words, $\operatorname{dist}\left(\Theta, \Sigma_{r} y+\Gamma\right)=$ 0 and, according to (2.23), $L(y, \theta)=x$.

Note. The map $L$ defined by (2.24) has the property

$$
L(y, \theta) \in \Theta, \quad \forall y .
$$

Proof of Theorem 2.11. Consider

$$
F^{l}(y, \theta):=\Delta^{r} \circ P^{l} \circ L(y, \theta)
$$

where $L: \mathbb{R}^{R-r} \times \mathbb{P}\left(\mathbb{R}^{R}\right) \rightarrow \mathbb{R}^{R}$ is defined by (the proof of) Lemma 2.12 . Note that $L$ is well defined since $R>r$.

If $\Delta^{r} x \neq 0$, then (2.20) holds because of (2.21), (2.26), and (2.19). On the other hand, if $\Delta^{r} x=0$, then by the $\Pi_{r-1}$ reproducing property of $S$, the left-hand side of (2.20) also vanishes so, by the definition of $L,(2.20)$ again holds in this case.

Saying that a map from a one- (or zero-)dimensional vector space to another vector space is homogeneous is no different from saying that it is linear. Since $P^{l}$ is homogeneous and $L_{\theta}:=L(\cdot, \theta)$ is, by (2.25), a rank $\leq 1$ linear map, $P^{l} \circ L_{\theta}$ and $\Delta^{r} \circ P^{l} \circ L_{\theta}=F^{l}$ are also linear maps with rank $\leq 1$.

Another elementary observation is the following refinement of Corollary 2.6.

Proposition 2.13. If $S$ is an affine invariant subdivision operator that reproduces $\Pi_{r}$ with $r>0$, then $S^{[1]}$ is a homogeneous subdivision operator that reproduces $\Pi_{r-1}$.

Putting together Theorem 2.5, Proposition 2.13, Lemma 2.8, and Theorem 2.11 we obtain the main tools for the development in the next section. We summarize these results in the order 2 case in Figure 2.

\section{Smoothness Analysis}

We present in this section some analysis results related to the smoothness of homogeneous subdivision schemes. 


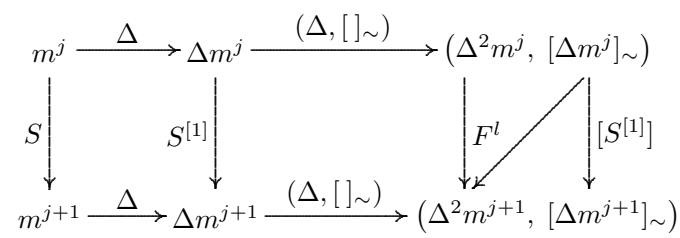

Fig. 2. Commutation relations for an order 2 affine invariant subdivision operator $S$. Note: $F^{l}$ are the maps obtained by applying Theorem 2.11 to $S^{[1]}$.

First we recall the definition of Hölder (a.k.a. $L^{\infty}$ Lipschitz) smoothness. If $\alpha=r+\beta$, $r \in \mathbb{N} \cup\{0\}$, and $0<\beta \leq 1$, then the Hölder space Lip $\alpha$ is the space of functions $f: \mathbb{R} \rightarrow \mathbb{R}$ with bounded derivatives of degree up to $r$, and

$$
|f|_{\text {Lip } \alpha}:=\sup _{x \neq y} \frac{\left|f^{(r)}(x)-f^{(r)}(y)\right|}{|x-y|^{\beta}}<\infty .
$$

\subsection{Known Results on Hölder Regularity of Subdivision}

The following is well known from the literature of linear subdivision schemes, see, e.g., [1], [14].

Theorem 3.1. Let $S=S_{Q}{ }^{1}$ be a linear subdivision operator of order $K$. Let $0<r \leq$ $K$. For $m \in \ell^{\infty}(\mathbb{Z})$, write $m_{j}:=S^{j} m$. Define

$$
\bar{v}_{r}(m):=\sup \left\{v:\left\|\Delta^{r} m_{j}\right\|_{\infty}=O\left(d^{-j v}\right)\right\} .
$$

If $\bar{v}_{r}(m)>0$, then the sequence of functions

$$
f_{j}:=\sum_{k \in \mathbb{Z}} m_{j, k} 1_{\left[d^{-j} k, d^{-j}(k+1)\right)}, \quad j=0,1,2, \ldots,
$$

converges uniformly to a bounded continuous function $f$; moreover,

$$
\sup \{\alpha: f \in \operatorname{Lip} \alpha\}=: v(f) \geq \bar{v}_{r}(m) .
$$

Remark. When $r>v(f)$ and under a stability condition, equality holds in (3.3).

Definition 3.2. We say that a subdivision operator $S$ is convergent if, for any $m \in \ell(\mathbb{Z})$, the sequence $f_{j}:=\sum_{k \in \mathbb{Z}} m_{j, k} 1_{\left[d^{-j} k, d^{-j}(k+1)\right)}, j=0,1,2, \ldots, m_{j}:=S^{j} m$, converges uniformly on compact sets to a limit function; we denote such a limit function by $S^{\infty} m$, which is necessarily unique if it exists. For a convergent subdivision operator $S$, we define its (Hölder) smoothness by

$$
s_{\infty}(S):=\inf _{m \in \ell^{\infty}} v\left(S^{\infty} m\right) .
$$

\footnotetext{
${ }^{1}$ The phase factor $n$ in $S_{Q, n}$ plays essentially no role in smoothness analysis. For instance, the convergence of $f_{j}$ in (3.2) is independent of $n$. For this reason, we shall drop the subscript $n$ unless there is a source of confusion.
} 
The proof of Theorem 3.1 is facilitated by (2.18) (hence the order $K$ condition) - a structure very specific to linear subdivision. An obvious open question would be: What is a natural generalization of this theorem when $Q$ is allowed to be nonlinear? While it seems that a certain direct generalization is possible_perhaps partly based on Theorem 2.11, we avoid the technical difficulty in facing this question by following the perturbation of linear scheme approach used in [12], [3]. For a given nonlinear subdivision operator $S$, this approach requires one to search for a suitable approximating linear subdivision operator $\bar{S}$.

Theorem 3.3 ([3, Theorem 3.3]). Let $S$ be a subdivision operator with dilation factor $d$ and let $\bar{S}$ be a linear subdivision operator with the same dilation factor that satisfies all the assumptions in Theorem 3.1 (of $S_{Q}$ there). If there exists a constant $C>0$ such that

$$
\left\|(S-\bar{S}) S^{j} m\right\|_{\infty} \leq C\|m\|_{\infty} d^{-j v}
$$

then $S$ is convergent and

$$
s_{\infty}(S) \geq \min \left(v, s_{\infty}(\bar{S})\right) .
$$

For specific median-interpolating subdivision operators $S_{Q}$ which are nonlinear and of order 2 (denoted as $S_{1,1,2}$ and $S_{1,1,3}$ in Section 5), Oswald established in [12], using explicit calculations based on available closed-form formulas for $Q$, the following interesting bound:

$$
\left\|(S-\bar{S}) S^{j} m\right\|_{\infty} \leq C\left\|\Delta^{2} S^{j} m\right\|_{\infty}
$$

where $\bar{S}$ is a linear subdivision operator based on midpoint interpolation (denoted as $\bar{S}_{1,1,2}$ and $\bar{S}_{1,1,3}$, respectively, in Section 5). Combined with Theorem 3.3, (3.5) gives essentially the same conclusion as what Theorem 3.1 would give had a nonlinear version of it existed: the convergence and smoothness of $S$ can be estimated from the decay rate of $\left\|\Delta^{2} S^{j} m\right\|_{\infty}$ as $j$ goes to infinity.

We describe in the next section a strategy for determining the decay rate of $\left\|\Delta^{r} S^{j} m\right\|_{\infty}$ for a $\Pi_{r-1}$ reproducing homogeneous subdivision operator $S$, based on the results developed in Section 2.1. By virtue of Proposition 2.13, this strategy can also be applied to $S^{[1]}$ when $S$ is affine invariant.

\subsection{Decay of $\left\|\Delta^{r} S^{j} m\right\|_{\infty}$}

We assume throughout in this section that, as in Theorem 2.11, $S$ is a homogeneous subdivision operator reproducing $\Pi_{r-1}$. Let $R=\max \left(R_{S}, r+1\right)$. Let

$$
F^{l}: \mathbb{R}^{R-r} \times \mathbb{P}\left(\mathbb{R}^{R}\right) \rightarrow \mathbb{R}^{R-r}, \quad l=1, \ldots, d,
$$

be the maps constructed in Theorem 2.11 and let $P^{l}: \mathbb{R}^{R} \rightarrow \mathbb{R}^{R}$ be the maps that satisfy (2.6). Since $S$ is homogeneous, so are $P^{l}$ and we can define

$$
\varrho_{l}:=\left[P^{l}\right]: \mathbb{P}\left(\mathbb{R}^{R}\right) \rightarrow \mathbb{P}\left(\mathbb{R}^{R}\right)
$$


based on Lemma 2.8. Next, define the maps of the shrinking factor by

$$
\varsigma_{l}\left([x]_{\sim}\right):= \begin{cases}\frac{\left\|\Delta^{r} P^{l}(x)\right\|_{\infty}}{\left\|\Delta^{r} x\right\|_{\infty}}=\frac{\left\|F^{l}\left(\Delta^{r} x,[x]_{\sim}\right)\right\|_{\infty}}{\left\|\Delta^{r} x\right\|_{\infty}}, & \text { if } \Delta^{r} x \neq 0, \quad l=1, \ldots, d . \\ 0, & \text { otherwise, }\end{cases}
$$

Notice that $\varsigma_{l}: \mathbb{P}\left(\mathbb{R}^{R}\right) \rightarrow[0, \infty)$ is well defined: the quotient in (3.7) depends only on $\theta:=[x] \sim$ as $P^{l}$ is homogeneous.

We are now ready to state the main observation in this section.

Theorem 3.4. Assume the notations above. Let $v>0$. The following two statements are equivalent:

(i) There exists a constant $C>0$ such that, for all $m \in \ell^{\infty}(\mathbb{Z})$,

$$
\left\|\Delta^{r} S^{j} m\right\|_{\infty} \leq C\|m\|_{\infty} d^{-j v}, \quad \forall j .
$$

(ii) There exists a constant $C^{\prime}>0$ such that for any $\theta \in \mathbb{P}\left(\mathbb{R}^{R}\right)$ and for any sequence $\left(\varepsilon_{i}\right)_{i=1}^{\infty}, \varepsilon_{i} \in\{1, \ldots, d\}$, if we define $\theta_{0}:=\theta$,

$$
\theta_{j}:=\varrho_{\varepsilon_{j}}\left(\theta_{j-1}\right), \quad j=1,2, \ldots,
$$

then

$$
\varsigma_{\varepsilon_{j}}\left(\theta_{j-1}\right) \cdots \varsigma_{\varepsilon_{1}}\left(\theta_{0}\right) \leq C^{\prime} d^{-j v}, \quad \forall j .
$$

Proof. We only spell out in detail the proof for (ii) $\Rightarrow$ (i), proving the converse direction is just a matter of reversing the steps below.

For any given $m \in \ell^{\infty}(\mathbb{Z})$ and $j \in \mathbb{N}$, let $x^{j}$ be an arbitrary length $R$ segment of $S^{j} m$, i.e., $x^{j}=\left(S^{j} m\right)_{i}^{[R]}$ for some $i \in \mathbb{Z}$. It suffices to prove that there exists a constant $C>0$ independent of $m, j$, and $i$ such that

$$
\left\|\Delta^{r} x^{j}\right\|_{\infty} \leq C\|m\|_{\infty} d^{-j \nu} .
$$

It follows from Proposition 2.2 that, for $k=j, \ldots, 1$, there exist a (unique) length $R$ segment $x^{k-1}$ of $S^{k-1} m$ and a (unique) $\varepsilon_{k} \in\{1, \ldots, d\}$ such that

$$
x^{k}=P^{\varepsilon_{k}}\left(x^{k-1}\right) \text {. }
$$

Let $\theta_{0}:=\left[x^{0}\right] \sim$ and $\theta_{k}:=\varrho_{\varepsilon_{k}}\left(\theta_{k-1}\right)$, for $k=1, \ldots, j$. By the definition of $\varrho_{l}, \theta_{k}=\left[x^{k}\right]_{\sim}$.

If $\left\|\Delta^{r} x^{j}\right\|_{\infty}=0$, then (3.11) is obviously true. Otherwise, according to Proposition 2.10, $\left\|\Delta^{r} x^{k-1}\right\|_{\infty} \neq 0$ for all $k=j, \ldots, 1$, and

$$
\frac{\left\|\Delta^{r} x^{k}\right\|_{\infty}}{\left\|\Delta^{r} x^{k-1}\right\|_{\infty}}=\varsigma_{\varepsilon_{k}}\left(\theta_{k-1}\right)
$$

So

$$
\frac{\left\|\Delta^{r} x^{j}\right\|_{\infty}}{\left\|\Delta^{r} x^{0}\right\|_{\infty}}=\prod_{k=1}^{j} \frac{\left\|\Delta^{r} x^{k}\right\|_{\infty}}{\left\|\Delta^{r} x^{k-1}\right\|_{\infty}}=\varsigma_{\varepsilon_{j}}\left(\theta_{j-1}\right) \cdots \varsigma_{\varepsilon_{1}}\left(\theta_{0}\right) \leq C^{\prime} d^{-j v}
$$

and

$$
\left\|\Delta^{r} x^{j}\right\|_{\infty} \leq C^{\prime}\left\|\Delta^{r} x^{0}\right\|_{\infty} d^{-j v} \leq C^{\prime}(r+1)\left\|x^{0}\right\|_{\infty} d^{-j v} \leq C^{\prime}(r+1)\|m\|_{\infty} d^{-j v} .
$$

So (3.11) is proved with $C:=C^{\prime}(r+1)$. (The same constant also works for (3.8).) 


\section{Median-Interpolating Subdivision}

In this section we consider the nonlinear subdivision operator $S_{Q}$ first constructed in [16]. This is an affine invariant operator with dilation factor $d=3$, neighborhood factor $D=3$, phase factor $n=2$, and $Q: \mathbb{R}^{3} \rightarrow \mathbb{R}^{3}$ defined by

$$
Q\left(m_{1}, m_{2}, m_{3}\right):=\left[\operatorname{median}\left(\pi \mid\left[0, \frac{1}{3}\right]\right), \operatorname{median}\left(\pi \mid\left[\frac{1}{3}, \frac{2}{3}\right]\right), \operatorname{median}\left(\pi \mid\left[\frac{2}{3}, 1\right]\right)\right],
$$

where $\pi$ is the unique quadratic polynomial that satisfies median $(\pi \mid[i-2, i-1])=m_{i}$, $i=1,2,3$.

In this case, the map $Q$ possesses the following closed-form expression [4, Proposition 2.3]:

$$
Q\left(m_{1}, m_{2}, m_{3}\right)= \begin{cases}m_{1}+\left(m_{2}-m_{1}\right)\left(q_{1}(r), q_{2}(r), q_{3}(r)\right), & \text { if } m_{1} \neq m_{2}, \\ \left(\frac{10}{9} m_{2}-\frac{1}{9} m_{3}, m_{2}, \frac{7}{9} m_{2}+\frac{2}{9} m_{3}\right), & \text { if } m_{1}=m_{2},\end{cases}
$$

where $r=\left(m_{3}-m_{2}\right) /\left(m_{2}-m_{1}\right)$ and $q_{1}, q_{2}, q_{3}: \mathbb{R} \rightarrow \mathbb{R}$ are defined by

$$
\begin{aligned}
& q_{1}(r)= \begin{cases}\frac{59}{27}+\frac{7}{27} r-\frac{8}{27} \sqrt{16+16 r+r^{2}}, & r \in\left[\frac{7}{3}, 5\right], \\
\frac{26}{27}+\frac{16}{27} r-\frac{4}{27} \sqrt{1+16 r+16 r^{2}}, & r \in\left[\frac{1}{5}, \frac{3}{7}\right], \\
\frac{77}{135}+\frac{13}{135} r+\frac{8}{135} \sqrt{1-62 r+r^{2}}, & r \in\left[-3,-\frac{1}{3}\right], \\
\frac{323-214 r+35 r^{2}}{288(1-r)}, & r \in[-11,-3), \\
\frac{7}{9}-r / 9, & \text { otherwise, }\end{cases} \\
& q_{2}(r)= \begin{cases}\frac{1097-1174 r+17 r^{2}+(278-8 r) \sqrt{1-62 r+r^{2}}}{270\left(4-4 r+\sqrt{1-62 r+r^{2}}\right)} & r \in\left[-\frac{10}{7},-\frac{7}{10}\right], \\
\frac{23}{30}+\frac{7}{30} r+\frac{1}{15} \sqrt{1-62 r+r^{2}}, & r \in\left[-3,-\frac{1}{3}\right] \backslash\left[-\frac{10}{7},-\frac{7}{10}\right], \\
1, & \text { otherwise, }\end{cases} \\
& q_{3}(r)= \begin{cases}1+r-r q_{1}(1 / r), & \text { if } r \neq 0, \\
\lim _{r \rightarrow 0} q_{3}(r)=\frac{10}{9}, & \text { if } r=0 .\end{cases}
\end{aligned}
$$

Let $S_{Q_{L}}$ be the linear subdivision operator with the same locality, dilation, and phase factor as $S_{Q}$, except that $Q$ is replaced by the following linear map:

$$
Q_{L}:=\left(\begin{array}{ccc}
\frac{2}{9} & \frac{8}{9} & -\frac{1}{9} \\
0 & 1 & 0 \\
-\frac{1}{9} & \frac{8}{9} & \frac{2}{9}
\end{array}\right) .
$$

Note that, for $m \in \mathbb{R}^{3}$,

$Q(m)=Q_{L}(m)$ if $m_{1}=m_{2}$ or $\left(m_{3}-m_{2}\right) /\left(m_{2}-m_{1}\right) \notin\left(-11,-\frac{1}{11}\right) \cup\left(\frac{1}{5}, \frac{3}{7}\right) \cup\left(\frac{7}{3}, 5\right)$.

In the rest of this section we prove the following result conjectured in [16], [4]: 
Proposition 4.1. $s_{\infty}\left(S_{Q}\right)=s_{\infty}\left(S_{Q_{L}}\right)=1$.

Proof. $1^{\circ}$. It has been shown in [12] that there exists a constant $C>0$ such that

$$
\left\|\left(Q-Q_{L}\right) m\right\|_{\infty} \leq C\left\|\Delta^{2} m\right\|_{\infty}
$$

and therefore the bound (3.5) holds with $S=S_{Q}$ and $\bar{S}=S_{Q_{L}}$. By standard techniques in linear subdivision one can verify that $s_{\infty}\left(S_{Q_{L}}\right)=1$ [16], [4]. Thus, by (3.5) and Theorem 3.3, if we can show that

$$
\left\|\Delta^{2} S^{j} m\right\|_{\infty} \leq C_{S}\|m\|_{\infty} 3^{-j}
$$

for some constant $C_{S}>0$ independent of $m$, then we have $s_{\infty}\left(S_{Q}\right) \geq 1$.

One way to show that $s_{\infty}\left(S_{Q}\right) \leq 1$ is to use the converse part of [4, Theorem 3.4], which states that if, for $S=S_{Q}$ and arbitrary $m \in \ell^{\infty}$, the sequence of functions (3.2) converges uniformly to a function in Lip 1 , then

$$
\left\|\Delta S_{Q}^{j} m\right\|_{\infty}=O\left(3^{-j}\right)
$$

for any fixed $m \in \ell^{\infty}$. We now show that (4.6) cannot hold for all $m$.

It suffices to show that $\left\|\Delta\left(S_{Q}^{j} m\right)_{-2}^{[4]}\right\|_{\infty}=O\left(3^{-j}\right)$ does not hold. Choose a sequence $m$ such that $m_{-2}^{[4]}=(0,0,1,1)$, then $\left[\Delta m_{-2}^{[4]}\right]_{\sim}=[(0,1,0)]_{\sim}$. By Lemma 6.2 below, $\left[\Delta\left(S_{Q}^{j} m\right)_{-2}^{[4]}\right] \sim \in L\left(=\left\{[x, 1, y] \sim:(x, y) \in\left[-\frac{1}{11}, 1\right)^{2}\right\}\right)$ for all $j$ and hence

$$
\left(S_{Q^{j}}^{j}\right)_{-2}^{[4]}=\left(S_{Q_{L}}^{j} m\right)_{-2}^{[4]}, \quad \forall j=0,1, \ldots
$$

A standard calculation in linear subdivision (applied to $S_{Q_{L}}^{[1]}$ ) gives

$$
\Delta\left(S_{Q_{L}}^{j} m\right)_{-2}^{[4]}=\left[\begin{array}{ccc}
\frac{1}{9} & \frac{2}{9} & 0 \\
-\frac{1}{9} & \frac{5}{9} & -\frac{1}{9} \\
0 & \frac{2}{9} & \frac{1}{9}
\end{array}\right]^{j}\left[\begin{array}{l}
0 \\
1 \\
0
\end{array}\right] \Rightarrow\left\|\Delta\left(S_{Q_{L}}^{j} m\right)_{-2}^{[4]}\right\|_{\infty} \asymp j 3^{-j} \neq O\left(3^{-j}\right) .
$$

Thus it remains to show (4.5), for this purpose we use Theorem 3.4.

$2^{\circ} . S=S_{Q}$ is an order 2 affine invariant subdivision operator with $R_{S}=4$ and $R_{S^{[1]}}=3$. Let $m \in \mathbb{R}^{4}$, then

$$
S(m)=\left\{\begin{array}{r}
\left(\frac{2}{9} m_{1}+\frac{7}{9} m_{2}, m_{2}, \frac{10}{9} m_{2}-\frac{1}{9} m_{1}, \frac{10}{9} m_{2}-\frac{1}{9} m_{4}, m_{2}, \frac{7}{9} m_{2}+\frac{2}{9} m_{4}\right), \\
\text { if } m_{2}=m_{3}, \\
\left(m_{3}-\left(m_{3}-m_{2}\right)\left(q_{3}(x), q_{2}(x), q_{1}(x)\right), m_{2}+\left(m_{3}-m_{2}\right)\left(q_{1}(y), q_{2}(y), q_{3}(y)\right)\right), \\
\text { otherwise, }
\end{array}\right.
$$

where $x=\left(m_{2}-m_{1}\right) /\left(m_{3}-m_{2}\right)$ and $y=\left(m_{4}-m_{3}\right) /\left(m_{3}-m_{2}\right)$. Since $S^{[1]} \circ \Delta=$ 
$\Delta \circ S$, by substituting $d_{i}=m_{i+1}-m_{i}, i=1,2,3$, into (4.7), we get

(4.8)

$$
\begin{aligned}
& S^{[1]}\left(d_{1}, d_{2}, d_{3}\right) \\
& \quad=\left\{\begin{array}{rr}
\frac{1}{9}\left(2 d_{1}, d_{1},-\left(d_{1}+d_{3}\right), d_{3}, 2 d_{3}\right), & \text { if } d_{2}=0, \\
d_{2}\left(q_{3}(x)-q_{2}(x), q_{2}(x)-q_{1}(x), q_{1}(x)+q_{1}(y)-1, q_{2}(y)-q_{1}(y), q_{3}(y)-q_{2}(y)\right), & \text { otherwise }
\end{array}\right.
\end{aligned}
$$

where $x=d_{1} / d_{2}$ and $y=d_{3} / d_{2}$.

Let $\varrho_{i}: \mathbb{P}\left(\mathbb{R}^{3}\right) \rightarrow \mathbb{P}\left(\mathbb{R}^{3}\right), i=1,2,3$, be the maps defined by applying (3.6) to the homogeneous $S^{[1]}$. Since each element in $\mathbb{P}\left(\mathbb{R}^{3}\right)$ can be represented as either $[(x, 0, y)] \sim$ or $[(x, 1, y)]_{\sim}$, by $(4.8) \varrho_{i}$ admit the following closed-form expressions:

$\varrho_{1}(\theta)= \begin{cases}{[(2 x, x,-x-y)]_{\sim},} & \text { if } \theta=[(x, 0, y)]_{\sim}, \\ {\left[\left(q_{3}(x)-q_{2}(x), q_{2}(x)-q_{1}(x), q_{1}(x)+q_{1}(y)-1\right)\right]_{\sim},} & \text { if } \theta=[(x, 1, y)]_{\sim},\end{cases}$

$\varrho_{2}(\theta)= \begin{cases}{[(x,-x-y, y)]_{\sim},} & \text { if } \theta=[(x, 0, y)]_{\sim}, \\ {\left[\left(q_{2}(x)-q_{1}(x), q_{1}(x)+q_{1}(y)-1, q_{2}(y)-q_{1}(y)\right)\right]_{\sim},} & \text { if } \theta=[(x, 1, y)]_{\sim},\end{cases}$

$\varrho_{3}(\theta)= \begin{cases}{[(-x-y, y, 2 y)]_{\sim},} & \text { if } \theta=[(x, 0, y)]_{\sim}, \\ {\left[\left(q_{1}(x)+q_{1}(y)-1, q_{2}(y)-q_{1}(y), q_{3}(y)-q_{2}(y)\right)\right]_{\sim},} & \text { if } \theta=[(x, 1, y)]_{\sim} .\end{cases}$

Define $\varsigma_{l}$ by applying (3.7) to $S^{[1]}$ with $r=1$ and let $\varsigma: \mathbb{P}\left(\mathbb{R}^{3}\right) \rightarrow[0, \infty)$ be defined by $\varsigma(\theta):=\max _{l=1,2,3} \varsigma_{l}(\theta)$. Then, by (4.8),

$$
\varsigma(\theta)= \begin{cases}\frac{1}{9} \frac{\max (|x|,|2 x+y|,|x+2 y|,|y|)}{\max (|x|,|y|)}, & \text { if } \theta=[x, 0, y] \sim \text { and }(x, y) \neq(0,0), \\ \frac{\max (D(x, y), D(y, x))}{\max (|x-1|,|y-1|)}, & \text { if } \theta=[x, 1, y] \sim \text { and }(x, y) \neq(1,1), \\ 0, & \text { otherwise, }\end{cases}
$$

where

$$
D(x, y):=\max \left(\left|q_{1}(x)-2 q_{2}(x)+q_{3}(x)\right|,\left|2 q_{1}(x)+q_{1}(y)-q_{2}(x)-1\right|\right) .
$$

One can verify that $\varsigma([x, 0, y] \sim)=\lim _{c \rightarrow \infty} \varsigma([c x, 1, c y] \sim)$ when $(x, y) \neq(0,0)$. We also abuse notation and write

$$
\varsigma(x, y):=\varsigma([x, 1, y] \sim) .
$$




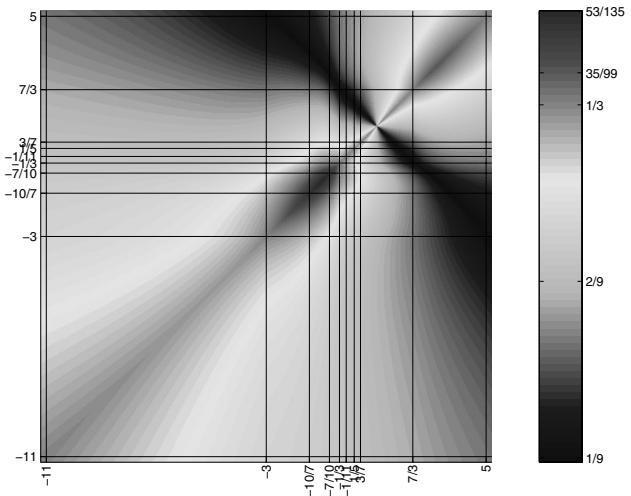

(a)

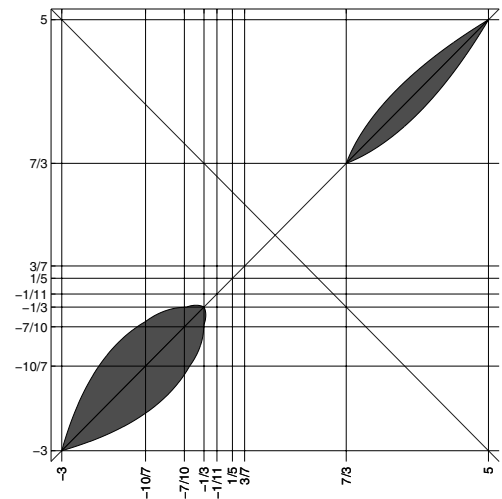

(b)

Fig. 3. (a) Shrinking factor $\varsigma(x, y)$. (b) Bad zone $\mathcal{B}=\left\{(x, y): \varsigma(x, y)>\frac{1}{3}\right\} \subset B_{1} \cup B_{2}$. These figures are produced by using (4.12) and (4.2), based on dividing the $(x, y)$ plane into $11 \times 11$ rectangles $I \times J$, where $I$ and $J$ are one of $(-\infty,-11],[-11,-3],\left[-3,-\frac{10}{7}\right],\left[-\frac{10}{7},-\frac{7}{10}\right],\left[-\frac{7}{10},-\frac{1}{3}\right],\left[-\frac{1}{3},-\frac{1}{11}\right],\left[-\frac{1}{11}, \frac{1}{5}\right],\left[\frac{1}{5}, \frac{3}{7}\right]$, $\left[\frac{3}{7}, \frac{7}{3}\right],\left[\frac{7}{3}, 5\right],[5, \infty)$.

By Theorem 3.4, to prove the bound (4.5) it suffices to show that there exists an absolute constant $C>0$ such that for any $\theta \in \mathbb{P}\left(\mathbb{R}^{3}\right)$ and any sequence $\left(\varepsilon_{i}\right)_{i=1}^{\infty}, \varepsilon_{i} \in\{1,2,3\}$, if we define

$$
\theta_{0}:=\theta, \quad \theta_{j}:=\varrho_{\varepsilon_{j}}\left(\theta_{j-1}\right)
$$

then, for any $j \geq 1$,

$$
\prod_{k=0}^{j-1} \varsigma\left(\theta_{k}\right) \leq C 3^{-j}
$$

$3^{\circ}$. If $\varsigma(\theta) \leq \frac{1}{3}$ for all $\theta$, then (4.15) holds for a trivial reason; unfortunately the "bad zone"

$$
\mathcal{B}:=\left\{\theta \in \mathbb{P}\left(\mathbb{R}^{3}\right): \varsigma(\theta)>\frac{1}{3}\right\}
$$

is nonempty. (See also Figure 3.)

Consider the partition $\mathbb{P}\left(\mathbb{R}^{3}\right)=B \cup G$ where

$$
B:=\left\{\left[\left(a_{1}, a_{2}, a_{3}\right)\right]_{\sim} \in \mathbb{P}\left(\mathbb{R}^{3}\right):\left(a_{1}-a_{2}\right)\left(a_{3}-a_{2}\right)>0\right\}
$$

and

$$
G:=\left\{\left[\left(a_{1}, a_{2}, a_{3}\right)\right]_{\sim} \in \mathbb{P}\left(\mathbb{R}^{3}\right):\left(a_{1}-a_{2}\right)\left(a_{3}-a_{2}\right) \leq 0\right\} .
$$

Let

$$
\begin{array}{ll}
B_{1}:=\left\{[(x, 1, y)]_{\sim}:(x, y) \in\left[\frac{7}{3}, 5\right]^{2}\right\}, & B_{2}:=\left\{[(x, 1, y)]_{\sim}:(x, y) \in\left[-3,-\frac{1}{11}\right)^{2}\right\}, \\
B_{3}:=\left\{[(x, 1, y)]_{\sim}: x, y<1\right\}, & L:=\left\{[(x, 1, y)]_{\sim}:(x, y) \in\left[-\frac{1}{11}, 1\right)^{2}\right\} .
\end{array}
$$

Note that $L \subset B_{3} \subset B \subset \mathbb{P}\left(\mathbb{R}^{3}\right), B_{1}, B_{2} \subset B$. 
In the Appendix, Sections 6.1 and 6.2, we shall prove the following facts:

[D1] (i) $\varrho_{i}$ (B) $\subset G$ for $i=1,3$; and (ii) $\varrho_{2}(B) \subset B$.

[D2] For any $\theta \in \mathbb{P}\left(\mathbb{R}^{3}\right), \operatorname{orbit}\left(\theta, \varrho_{2}\right):=\left\{\varrho_{2}^{n}(\theta): n=0,1, \ldots\right\}$ satisfies

$$
\left|\operatorname{orbit}\left(\theta, \varrho_{2}\right) \cap B_{i}\right| \leq 1, \quad i=1,2 .
$$

[S1] $\mathcal{B} \subset B_{1} \cup B_{2} \subset B$.

[S2] $\max _{\theta \in B_{1}} \varsigma(\theta)=\varsigma\left(\frac{16}{5}, \frac{16}{5}\right)=\frac{35}{99}\left(>\frac{1}{3}\right)$.

[S3] $\max _{\theta \in B_{2}} \varsigma(\theta)=\varsigma(-1,-1)=\frac{53}{135}\left(>\frac{1}{3}\right)$.

[S4] $\max _{\theta \in G} \varsigma(\theta)=\varsigma( \pm 1, \mp 1)=\frac{34}{135}\left(<\frac{1}{3}\right)$.

The above facts combine to yield (4.15) with $C=C^{*}:=\frac{35}{99} \times \frac{53}{135} \times 9$, a key ingredient of this final step is that the "good factor" in [S4] is good enough to "compensate" the two "bad factors" in [S2] and [S3]: $\frac{35}{99} \times \frac{53}{135} \times \frac{34}{135}<\left(\frac{1}{3}\right)^{3}$.

$4^{\circ}$. In detail, the compensation argument goes as follows: Let $\theta \in \mathbb{P}\left(\mathbb{R}^{3}\right), \varepsilon_{i} \in$ $\{1,2,3\}, i=1,2, \ldots$, and let $\left(\theta_{n}\right)_{n=0}^{\infty}$ be defined by (4.14). Let $j \geq 1$.

To prove (4.15) holds for $C=C^{*}$ it suffices to show either:

(i) Equation (4.15) holds for $C=C^{*}$; or

(ii) $\exists j^{\prime}, 1 \leq j^{\prime} \leq j$, such that (4.15) holds for $j=j^{\prime}$ and $C=1$, i.e., $\prod_{k=0}^{j^{\prime}-1} \varsigma\left(\theta_{k}\right) \leq$ $3^{-j^{\prime}}$

it is because $\theta, j$, and $\varepsilon_{i}$ in the above are all arbitrary, so one can cycle the "reduction step" in (ii) as many times as needed in order to establish (4.15).

If $\theta_{i} \notin \mathcal{B}, \forall i$, then $\prod_{k=0}^{j-1} \varsigma\left(\theta_{k}\right) \leq 3^{-j}, \forall j$; otherwise, let $n \geq 0$ be the smallest index such that $\theta_{n} \in \mathcal{B}$.

If $\varepsilon_{n^{\prime}}=2$ for all $n^{\prime}>n$, then, by [D2] and [S1]-[S3], we have

$$
\prod_{k=0}^{j-1} \varsigma\left(\theta_{k}\right) \leq\left\{\begin{array}{ll}
3^{-j}, & \text { if } j \leq n, \\
3^{-(j-1)} \times \frac{53}{135}, & \text { if } j=n+1, \\
3^{-(j-2)} \times \frac{53}{135} \times \frac{35}{99}, & \text { if } j>n+1,
\end{array}\right\} \leq C^{*} 3^{-j}
$$

So (i) holds.

Otherwise let $m$ be the smallest index such that $m>n$ and $\varepsilon_{m} \neq 2$. Then $\mid\left\{\theta_{i}\right.$ : $i<n\} \cap \mathcal{B}|=0|,\left\{\theta_{i}: n \leq i \leq m-1\right\} \cap \mathcal{B} \mid \leq 2$ by [D2] and [S1]. By [D1](ii), $\theta_{m-1} \in B$, then $\theta_{m}=\varrho_{\varepsilon_{m}}\left(\theta_{m-1}\right) \in G$ by [D1](i). So either $j \leq m$, in this case (i) holds: $\prod_{k=0}^{j-1} \varsigma\left(\theta_{k}\right) \leq 3^{-(j-2)} \times \frac{53}{135} \times \frac{35}{99} \leq C^{*} 3^{-j}$, or $j>m$, in this case

$$
\prod_{k=0}^{m} \varsigma\left(\theta_{k}\right) \leq 3^{-(m-2)} \times \frac{35}{99} \times \frac{53}{135} \times \frac{34}{135}<3^{-(m+1)},
$$

and (ii) holds with $j^{\prime}=m+1$. 
Remark. [S1]-[S3] alone give (4.5) with the right-hand side replaced by the suboptimal $O\left(3^{-j \alpha}\right), \alpha=\log _{3}\left(\frac{135}{53}\right)$, which in turn gives $s_{\infty}\left(S_{Q}\right) \geq \log _{3}\left(\frac{135}{53}\right)$. This recovers what Oswald obtained in [12].

\section{A Linearization Heuristic}

Although this paper identifies (in Section 2) new general properties of nonlinear subdivision operators useful enough to determine the critical Hölder regularity of a nonlinear subdivision scheme, it is obvious that our current analysis tools are by no means satisfactory. Our proof of the Main Result (Proposition 4.1) is based not only on the general facts in Section 2 but also on a number of specific "tricky" calculations not supported by any general analysis method. For instance, a final step of our proof involves a highly specific analysis on the discrete dynamical behavior of three maps acting on the real projective plane $\mathbb{P}^{2}$. A richer collection of analysis tools for nonlinear subdivision is more desirable.

The development of a useful theory is usually guided by examples and applications. Therefore, in this section, we derive a large family of new conjectures of which the original conjecture proved in this paper is just a very special case. The derivation of this new set of conjectures is based on a linearization principle, which leads to a question of independent interest.

We consider the nonlinear subdivision operator $S_{\sigma, L, d}$ defined in (1.3)-(1.4), which includes median-interpolating subdivision as a very special case. We note that any $S_{\sigma, L, d}$ is offset invariant and that any $S_{p, L, d}:=S_{\left.|\cdot|\right|^{p}, L, d}$ is affine invariant (recall comments around (1.5) for the case of $p=\infty$ ). However, $S_{\sigma, L, d}$ is not homogeneous in general.

Motivated by Proposition 4.1 one may wonder if for any $S_{\sigma, L, d}$ there is a certain general linearization procedure giving in each case a linear subdivision operator $\bar{S}_{\sigma, L, d}$ which shares the same smoothness with $S_{\sigma, L, d}$.

A fundamental tool for finding a linear approximation to a nonlinear map is, of course, by taking derivatives. Recall from the informal discussion in Section 1.1 that our main insights as to why Proposition 4.1 should be true are: (i) the linearization approximation (1.6) exists for medians of monotone functions; and (ii) a smooth subdivision process tends to yield locally monotone data. While the notion of derivative does not seem to be in place in (1.6), we shall see that (1.6) can be interpreted as an action of taking a derivative after we address the following analysis question:

(Q) Is $m_{\sigma}: C(I) \rightarrow \mathbb{R}$ Gâteaux or Fréchet differentiable at a strictly monotone function $l \in C(I)$ ?

By the formal calculation in Section 6.3 of the Appendix, we see that if the Gâteaux derivative actually exists, then it is given by the following bounded positive linear functional on $C(I)$ :

$D m_{\sigma}(f ; l)=\int_{I} f(x) d \alpha(x), \quad \alpha(x):=\frac{\xi(x)}{\int_{I} d \xi(x)}, \quad \xi(x):=\int_{a}^{x} \frac{d \sigma^{\prime}\left(l(t)-m_{\sigma}(l)\right)}{l^{\prime}(t)}$. 


\subsection{Linearized $p$-Mean Subdivision $\bar{S}_{p, L, d}$}

For convenience, we write $m_{|\cdot|^{p}}=: m_{p}$, and extend the definition to $p=\infty$ by (1.2). We now specialize (5.1) to $m_{p}, p \in[1, \infty]$, and $l \in \Pi_{1} \backslash \Pi_{0}$, i.e., $l(x)=c(x-\bar{x})+c^{\prime}$, where $\bar{x}$ is the midpoint of $I=[a, b]$.

$p$-Mean for Nearly Straight Functions. For a nearly straight function $f(x) \approx l(x)=$ $c(x-\bar{x})+c^{\prime}$,

$$
\begin{aligned}
m_{p}(f) & \approx m_{p}(l)+D m_{p}(f-l ; l) \\
& =m_{p}(l)-D m_{p}(l, l)+D m_{p}(f ; l)=c^{\prime}-c^{\prime}+D m_{p}(f ; l) \\
(5.2) & =\int_{I} f(x) d \alpha_{p}(x), \text { where } \alpha_{p}(x)= \begin{cases}\frac{1}{2} \operatorname{sign}(x-\bar{x}), & p=1, \\
\frac{2^{p-2}}{|I|^{p-1}}|x-\bar{x}|^{p-1} \operatorname{sign}(x-\bar{x}), & 1<p<\infty, \\
\frac{1}{2} 1_{x=b}-\frac{1}{2} 1_{x=a}, & p=\infty,\end{cases} \\
(5.3) & = \begin{cases}f(\bar{x}), & p=1, \\
\int_{I} f(x) w_{p}(x) d x, \quad w_{p}(x)=2^{p-2}(p-1)|x-\bar{x}|^{p-2} /|I|^{p-1}, & 1<p<\infty, \\
\frac{1}{2}(f(a)+f(b)), & p=\infty,\end{cases} \\
(5.4) & =: \bar{m}_{p}(f)=: \bar{m}_{p}(f ; I) .
\end{aligned}
$$

We can now define, for $1 \leq p \leq \infty, \bar{S}_{p, L, d}$ based on replacing $m(; \sigma, I)$ in (1.3)-(1.4) by $\bar{m}_{p}(\cdot ; I)$. It is elementary to check that, as in the nonlinear case, $h>0$ plays no role in the definition, the interpolation problem in (1.3) is a well-posed linear problem, and $\bar{S}_{p, L, d}$ is a linear subdivision operator with dilation factor $d$.

We calculate the masks $\left(a_{i}\right)_{i \in \mathbb{Z}}$ of the linear subdivision operators $\bar{S}_{p, L, d}$ for $L=1,2$ and $d=2,3$ : by (2.3), we see that the phase and locality factors of $\bar{S}_{p, L, d}$ are $n=L+1$ and $D=2 L+1$, respectively; by (2.4) one sees that the mask of $\bar{S}_{p, L, d}$ is supported at $[-L d,(L+1) d-1]$, and, by symmetry, $a_{i}=a_{2 L d+d-1}$. Below, we write the masks in $z$-domain: $\widehat{a}(z):=\sum_{i=-L d}^{(L+1) d-1} a_{i} z^{i}$. It can be shown that $\bar{S}_{p, L, d}$ is of order $2 L+1$, and, hence, $\widehat{a}(z)$ possesses the factor $\left(1+z+\cdots+z^{d-1}\right)^{2 L+1}$; moreover, the subdivision operator $\bar{S}_{p, L, d}^{[2 L+1]}$ (of dilation factor $d$ ) with mask $\widehat{b}(z)=\widehat{a}(z) /\left(1+z+\cdots+z^{d-1}\right)^{2 L+1}$ satisfies $\bar{S}_{p, L, d}^{[2 L+1]} \circ \Delta^{2 L+1}=\Delta^{2 L+1} \circ \bar{S}_{p, L, d}$.

$$
\begin{aligned}
& \underline{L=1, d=2:} \quad \widehat{a}(z)=(1+z)^{3}\left(-3 p+(4+10 p) z-3 p z^{2}\right) \frac{z^{-2}}{16(1+p)} . \\
& \underline{L=1, d=3:} \quad \widehat{a}(z)=\left(1+z+z^{2}\right)^{3}\left(-2 p+(5 p+1) z-2 p z^{2}\right) \frac{z^{-3}}{9(p+1)} . \\
& \underline{L=2, d=2:} \quad \widehat{a}(z)=(1+z)^{5}\left(b_{0} z^{4}+b_{1} z^{3}+b_{2} z^{2}+b_{1} z+b_{0}\right) \frac{z^{-4}}{256(p+3)(p+1)^{2}}, \\
& b_{0}=11 p^{3}+37 p^{2}+12 p+10, \quad b_{1}=-60 p^{3}-216 p^{2}-112 p-52, \\
& b_{2}=114 p^{3}+438 p^{2}+312 p+132 \text {. }
\end{aligned}
$$




$$
\begin{gathered}
\frac{L=2, d=3: \quad \widehat{a}(z)=\left(1+z+z^{2}\right)^{5}\left(b_{0}+b_{1} z+b_{2} z^{2}+b_{1} z^{3}+b_{0} z^{4}\right) \frac{z^{-6}}{486(p+3)(p+1)^{2}},}{b_{0}=26 p^{3}+87 p^{2}+27 p+20, \quad b_{1}=-120 p^{3}-416 p^{2}-172 p-92} \\
b_{2}=194 p^{3}+688 p^{2}+332 p+162 .
\end{gathered}
$$

\subsection{One Down (an Infinite) More to Go}

Under the new notations, Proposition 4.1 can be rewritten as $s_{\infty}\left(S_{1,1,3}\right)=s_{\infty}\left(\bar{S}_{1,1,3}\right)$. In this section we provide computational evidence for the conjecture that the natural question

$$
s_{\infty}\left(S_{p, L, d}\right) \stackrel{?}{=} s_{\infty}\left(\bar{S}_{p, L, d}\right)
$$

may well have an affirmative answer for at least many more different values of $1 \leq p \leq$ $\infty, L \geq 1$, and $d \geq 2$.

Of course the above conjecture is true for $p=2$, as 2-mean is a linear functional, so $m_{|\cdot|^{2}}=\bar{m}_{|\cdot|^{2}}$ and $S_{2, L, d}=\bar{S}_{2, L, d}$. We have proved in Proposition 4.1 that (5.5) is true for $(p, L, d)=(1,1,3)$. In [15], (5.5) is proved for $(p, L, d)=(\infty, 1,3)$.

By Theorem (3.1), one can conclude that

$$
s_{\infty}\left(\bar{S}_{p, L, d}\right) \geq-\log _{d} \rho\left(\bar{S}_{p, L, d}^{[2 L+1]}\right) .
$$

A standard stability result in the theory of the linear refinement equation can be applied to show that equality holds above, due to space constraints we avoid spelling out the technical details. Also in each case, one can verify that $\widehat{b}(z) /\left(1+z+\cdots+z^{d-1}\right)$ is nonnegative on the unit circle of the complex plane, this implies by a well-known positivity argument that the spectral radius of the operator $\bar{S}_{p, L, d}^{[2 L+1]}$ becomes reduced to the spectral radius of a finite matrix (see, e.g., [14]), ${ }^{2}$ and we get

$$
\begin{gathered}
\rho\left(\bar{S}_{p, 2,2}^{[5]}\right)=\frac{c_{0}+\sqrt{c_{1}}}{256(p+3)(p+1)^{2}}, \quad c_{0}=27 p^{3}+111 p^{2}+100 p+40, \\
c_{1}=6249 p^{6}+47706 p^{5}+123929 p^{4}+138840 p^{3}+94256 p^{2}+35520 p+7424 .
\end{gathered}
$$

Since the critical Hölder exponent of $S$ is governed by the asymptotic decay rate of $\left\|\Delta^{r} S^{j} v\right\|_{\ell^{\infty}}$ for a large enough differencing order $r$, we compare, in each case, the

\footnotetext{
${ }^{2}$ When $L=1, d=2,3$, or $L=2, d=3$, we even have $\rho\left(\bar{S}_{p, L, d}^{[2 L+1]}\right)=\left\|\bar{S}_{p, L, d}^{[2 L+1]}\right\|_{\ell \infty}$, but this is already not true when $L=2, d=2$.
} 


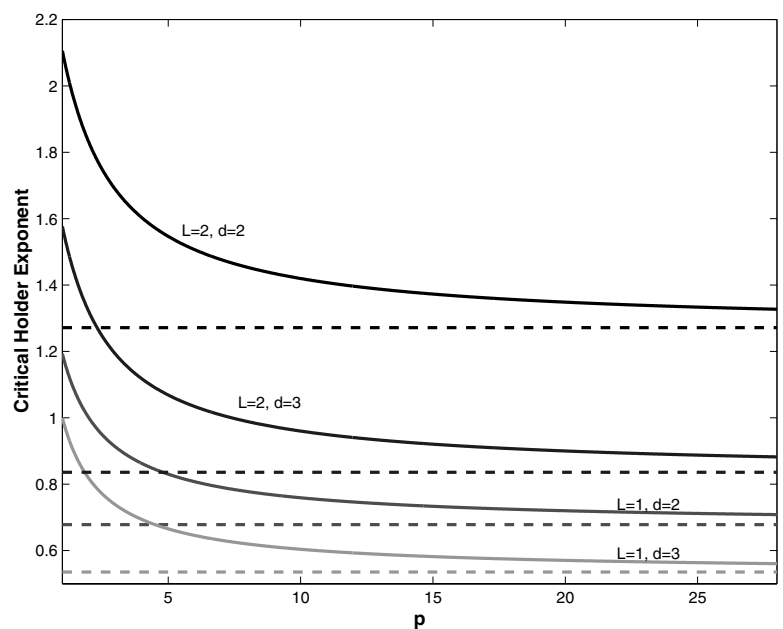

Fig. 4. Critical Hölder exponent of $\bar{S}_{p, L, d}$.

right-hand side of (5.6) with a computed

$$
s_{\infty}^{\text {Est. }}\left(S_{p, L, d}\right):=-\log _{d} \frac{\left\|\Delta^{r}\left(S_{p, L, d}\right)^{7} \delta\right\|_{\ell^{\infty}}}{\left\|\Delta^{r}\left(S_{p, L, d}\right)^{6} \delta\right\|_{\ell^{\infty}}}, \quad r=2 L \quad \text { or } \quad 2 L+1 .
$$

The nonlinear subdivision operator $S_{p, L, d}$ is implemented numerically using the algorithms proposed in [13].

Table 2. Numerical evidences for conjecture (5.5). In each case, the estimated smoothness $s_{\infty}^{\text {Est. }}\left(S_{p, L, d}\right)$ agrees with $s_{\infty}\left(\bar{S}_{p, L, d}\right)$ up to at least two to four significant digits.

\begin{tabular}{lcccccc}
\hline \multicolumn{5}{c}{$(L, d)=(1,2)$} \\
\hline & $-\log _{2} \rho\left(\bar{S}_{p, 1,2}^{[3]}\right)$ & $s_{\infty}^{\text {Est. }}, r=2$ & $s_{\infty}^{\text {Est. }}, r=3$ & $-\log _{3} \rho\left(\bar{S}_{p, 1,3}^{[3]}\right)$ & $s_{\infty}^{\text {Est. }}, r=2$ & $s_{\infty}^{\text {Est. }}, r=3$ \\
\hline$p=1.0$ & 1.1926 & 1.2038 & 1.1926 & 1.0000 & 1.0009 & 1.0000 \\
$p=1.2$ & 1.1375 & 1.1473 & 1.1375 & 0.9464 & 0.9471 & 0.9464 \\
$p=1.4$ & 1.0931 & 1.1020 & 1.0931 & 0.9041 & 0.9046 & 0.9041 \\
$p=1.6$ & 1.0566 & 1.0648 & 1.0565 & 0.8697 & 0.8701 & 0.8697 \\
$p=1.8$ & 1.0260 & 1.0338 & 1.0260 & 0.8413 & 0.8416 & 0.8413 \\
$p=\infty$ & 0.6781 & 0.6813 & 0.6781 & 0.5350 & 0.5282 & 0.5281 \\
\hline \multicolumn{7}{c}{$(L, d)=(2,2)$} \\
& $-\log _{2} \rho\left(\bar{S}_{p, 2,2}^{[5]}\right)$ & $s_{\infty}^{\text {Est. }}, r=4$ & $s_{\infty}^{\text {Est. }}, r=5$ & $-\log _{3} \rho\left(\bar{S}_{p, 2,3}^{[5]}\right)$ & $s_{\infty}^{\text {Est. }}, r=4$ & $s_{\infty}^{\text {Est. }}, r=5$ \\
\hline$p=1.0$ & 2.1056 & 2.1037 & 2.1049 & 1.5764 & 1.5764 & 1.5764 \\
$p=1.2$ & 2.0325 & 2.0333 & 2.0293 & 1.5072 & 1.5070 & 1.5070 \\
$p=1.4$ & 1.9701 & 1.9717 & 1.9658 & 1.4488 & 1.4485 & 1.4485 \\
$p=1.6$ & 1.9166 & 1.9182 & 1.9122 & 1.3992 & 1.3989 & 1.3989 \\
$p=1.8$ & 1.8704 & 1.8717 & 1.8664 & 1.3566 & 1.3564 & 1.3564 \\
$p=\infty$ & 1.2714 & 1.2670 & 1.2663 & 0.8359 & 0.8445 & 0.8463 \\
\hline
\end{tabular}


We comment that the three-parameter family of conjectures (5.5) can be extended in at least one more dimension. In linear subdivision, extensive results have been obtained is smoothness measured by $L^{p^{\prime}}$ instead of the uniform norm. Hölder smoothness corresponds to $p^{\prime}=\infty$ (hence the subscript in $s_{\infty}$ ), whereas the $p^{\prime}=2$ case enjoys a Hilbert space structure which, in particular, yields very useful computational results (see [8] and references therein). We end this paper with the following open question:

$$
s_{p^{\prime}}\left(S_{p, L, d}\right) \stackrel{?}{=} s_{p^{\prime}}\left(\bar{S}_{p, L, d}\right), \quad \forall p, p^{\prime} \in[1, \infty], \quad L \geq 1, \quad d \geq 2 .
$$

\section{References}

1. A. S. Cavaretta, W. Dahmen, C. A. Micchelli (1991): Stationary subdivision. Mem. Amer. Math. Soc., 453

2. A. Cohen, N. Dyn, B. MATEI (2003): Quasilinear subdivision schemes with applications to ENO interpolation. Appl. Comput. Harmon. Anal., 15(2):89-116.

3. I. DAUBeChiES, O. RUNBORG, W. SWELDENS (2004): Normal multiresolution approximation of curves. Constr. Approx., 3(4):399-463.

4. D. L. Donoho, T. P.-Y. YU (2000): Nonlinear pyramid transforms based on median-interpolation. SIAM J. Math. Anal., 31(5):1030-61.

5. M. S. FlOATER, C. A. MiCCHELLI (1998): Nonlinear stationary subdivision. In: Approximation Theory: In Memory of A. K. Varma (N. K. Govil, R. N. Mohapatra, Z. Nashed, A. Sharma, J. Szabados, eds.). New York: Marcel Dekker, pp. 209-224.

6. T. N. T. Goodman, T. P.-Y. YU (1999): Interpolation of medians. Adv. Comput. Math., 11(1):1-10

7. A. HARTEN (1993): Discrete multiresolution analysis and generalized wavelets. Appl. Numer. Math., 12:153-192.

8. R. Q. JIA, Q. T. JIANG (2003): Spectral analysis of the transition operator and its applications to smoothness analysis of wavelets. SIAM J. Matrix Anal. Appl., 24(4):1071-1109.

9. F. KuIJT, R. van DAMME (1998): Convexity preserving interpolatory subdivision schemes. Constr. Approx., 14:609-630.

10. J. L. Merrien, P. SABLONNIERE (2001): Monotone and convex $C^{1}$ Hermite interpolants generated by an adaptive subdivision scheme. C. R. Acad. Sci. Paris, Ser. I, Math., 333(5):493-497.

11. P. Oswald (2003): Smoothness of a nonlinear subdivision scheme. In: Curves and Surface Fitting: Saint-Malo 2002 (A. Cohen, J.-L. Merrien, L. L. Schumaker, eds.). Nashville, TN: Nashboro Press, pp. 323-332.

12. P. OsWALD (2004): Smoothness of nonlinear median-interpolation subdivision. Adv. Comput. Math., 20(4):401-423.

13. J. S. PANG, T. P.-Y. YU (2004): M-Estimators and their interpolation by polynomials. SIAM J. Numer. Anal., 42(3):997-1017.

14. O. Rioul (1992): Simple regularity criteria for subdivision schemes. SIAM J. Math. Anal., 23(6):15441576.

15. G. XIE, T. P.-Y. YU (2004): On a linearization principle for nonlinear p-mean subdivision schemes. In: Advances in Constructive Approximation (M. Neamtu, E. B. Saff, eds.). Nashville, TN: Nashboro Press, pp. 519-533.

16. T. P.-Y. YU (1997): New developments in interpolating wavelet transforms. PhD thesis. Program of Scientific Computing and Computational Mathematics, Stanford University, CA.

\section{Appendix}

\subsection{Dynamics of $\varrho_{i}$}

From (4.2), one can show by elementary calculus that

Lemma 6.1. $q_{1}, q_{1}-q_{2}$, and $-q_{1}+2 q_{2}-q_{3}$ are strictly decreasing. 
This lemma facilitates many of the calculations involved in the proof of [D1]-[D2] and [S1]-[S4].

Proof of [D1]. For part (i), by symmetry it suffices to prove the proposition for $i=1$ : if we define $\mathcal{R}: \mathbb{P}\left(\mathbb{R}^{3}\right) \rightarrow \mathbb{P}\left(\mathbb{R}^{3}\right)$ by $\mathcal{R}\left(\left[d_{1}, d_{2}, d_{3}\right]_{\sim}\right):=\left[d_{3}, d_{2}, d_{1}\right]_{\sim}$, then $\mathcal{R}(G)=G$, $\mathcal{R}(B)=B$, and $\varrho_{3} \circ \mathcal{R}=\mathcal{R} \circ \varrho_{1}$; thus if the proposition holds for $i=1$, then

$$
\varrho_{3}(B)=\varrho_{3}(\mathcal{R}(B))=\mathcal{R}\left(\varrho_{1}(B)\right) \subset \mathcal{R}(G)=G .
$$

Let $\theta=\left[\left(a_{1}, a_{2}, a_{3}\right)\right]_{\sim} \in B$. If $a_{2}=0$, then $a_{1} a_{3}>0$ and $\varrho_{1}(\theta)=\left[\left(2 a_{1}, a_{1},-a_{1}-\right.\right.$ $\left.\left.a_{3}\right)\right]_{\sim}$. Since $\left(2 a_{1}-a_{1}\right)\left(-a_{1}-a_{3}-a_{1}\right)=-2 a_{1}^{2}-a_{1} a_{3}<0,\left[\left(2 a_{1}, a_{1},-a_{1}-a_{3}\right)\right]_{\sim} \in G$.

If $a_{2} \neq 0$, then $\left[\left(a_{1}, a_{2}, a_{3}\right)\right]_{\sim}=[(x, 1, y)]_{\sim}$, where $x=a_{1} / a_{2}, y=a_{3} / a_{2}$. Since $[(x, 1, y)]_{\sim} \in B$, we have $(x-1)(y-1)>0$. Since $\varrho_{1}\left([(x, 1, y)]_{\sim}\right)=\left[\left(q_{3}(x)-\right.\right.$ $\left.\left.q_{2}(x), q_{2}(x)-q_{1}(x), q_{1}(x)+q_{1}(y)-1\right)\right]_{\sim}$, in order to prove $\varrho_{1}\left([(x, 1, y)]_{\sim}\right) \in G$, we need to show $\left(q_{1}(x)+q_{3}(x)-2 q_{2}(x)\right)\left(2 q_{1}(x)-q_{2}(x)+q_{1}(y)-1\right) \leq 0$.

By monotonicity, when $x>1$ and $y>1, q_{1}(x)+q_{3}(x)-2 q_{2}(x)>q_{1}(1)+q_{3}(1)-$ $2 q_{2}(1)=0$ and $2 q_{1}(x)-q_{2}(x)+q_{1}(y)-1<2 q_{1}(1)-q_{2}(1)+q_{1}(1)-1=0$; when $x<1$ and $y<1, q_{1}(x)+q_{3}(x)-2 q_{2}(x)<q_{1}(1)+q_{3}(1)-2 q_{2}(1)=0$ and $2 q_{1}(x)-q_{2}(x)+q_{1}(y)-1>2 q_{1}(1)-q_{2}(1)+q_{1}(1)-1=0$. Consequently, $\left(q_{1}(x)+q_{3}(x)-2 q_{2}(x)\right)\left(2 q_{1}(x)-q_{2}(x)+q_{1}(y)-1\right) \leq 0$ when $(x-1)(y-1)>0$, so $\varrho_{1}\left([(x, 1, y)]_{\sim}\right) \in G$.

Part [D1](ii) can be proved similarly, see also the following lemma.

Lemma 6.2. $\varrho_{2}$ maps $B, B_{3}$, and $L$ into itself.

Proof. By (4.10),

$$
\varrho_{2}\left([(x, 1, y)]_{\sim}\right)=\left[\left(q_{2}(x)-q_{1}(x), q_{1}(x)+q_{1}(y)-1, q_{2}(y)-q_{1}(y)\right)\right] \sim .
$$

If $\theta \in B_{3}$, then $\theta=[x, 1, y] \sim$ with $x, y<1$, consequently, monotonicity gives $q_{2}(x)-$ $q_{1}(x), q_{2}(y)-q_{1}(y)<q_{2}(1)-q_{1}(1)=\frac{1}{3}$ and $q_{1}(x)+q_{1}(y)-1>q_{1}(1)+q_{1}(1)-1=\frac{1}{3}$. Therefore $\left(q_{2}(x)-q_{1}(x)\right) /\left(q_{1}(x)+q_{1}(y)-1\right),\left(q_{2}(y)-q_{1}(y)\right) /\left(q_{1}(x)+q_{1}(y)-1\right)<1$ and $\varrho_{2}(\theta) \in B_{3}$. Thus $\varrho_{2}\left(B_{3}\right) \subset B_{3}$.

Similar calculations show that $\varrho_{2}(B) \subset B$ and $\varrho_{2}(L) \subset\{[x, 1, y] \sim:(x, y) \in$ $\left.\left[\frac{7}{19}, 1\right)^{2}\right\} \subset L$.

Proof of [D2]. To prove $\left|\operatorname{orbit}\left(\theta, \varrho_{2}\right) \cap B_{i}\right| \leq 1$, it suffices to show that if $\theta \in B_{i}$, then $\varrho_{2}^{n}(\theta) \notin B_{i}$ for all $n>0$.

Recall (6.1) and that $q_{2}-q_{1}$ is increasing and $q_{1}$ is decreasing.

$1^{\circ}$. Assume $\theta \in B_{1}$. We show that $\varrho_{2}(\theta) \notin B_{1}$ and $\varrho_{2}^{2}(\theta) \in B_{3}$. Then, since $B_{3} \cap B_{1}=$ $\emptyset$ and, by Lemma 6.2, $B_{3}$ is invariant under $\varrho_{2}$, we conclude that $\varrho_{2}^{n}(\theta) \notin B_{1}$ for all $n>0$.

Since $\theta \in B_{1}, \theta=[(x, 1, y)]_{\sim}$ with $x, y \geq \frac{7}{3}$. It follows that $q_{2}(x)-q_{1}(x), q_{2}(y)-$ $q_{1}(y) \geq q_{2}\left(\frac{7}{3}\right)-q_{1}\left(\frac{7}{3}\right)=\frac{13}{27}$ and $q_{1}(x)+q_{1}(y)-1 \leq q_{1}\left(\frac{7}{3}\right)+q_{1}\left(\frac{7}{3}\right)-1=\frac{1}{27}$.

There are three cases: $q_{1}(x)+q_{1}(y)-1$; (i) $\in\left(0, \frac{1}{27}\right]$; (ii) $=0$; and (iii) $<0$. 
Case (i): In this case, we have

$$
\frac{q_{2}(x)-q_{1}(x)}{q_{1}(x)+q_{1}(y)-1}, \frac{q_{2}(y)-q_{1}(y)}{q_{1}(x)+q_{1}(y)-1} \geq \frac{13}{27} / \frac{1}{27}=13 .
$$

So $\varrho_{2}(\theta)=[(a, 1, b)]_{\sim}$ for some $a \geq 13, b \geq 13$. Therefore, $\varrho_{2}(\theta) \notin B_{1}$. Moreover,

$$
\varrho_{2}^{2}(\theta)=\varrho_{2}([(a, 1, b)] \sim)=\left[\left(q_{2}(a)-q_{1}(a), q_{1}(a)+q_{1}(b)-1, q_{2}(b)-q_{1}(b)\right)\right] \sim .
$$

It follows from the monotonicity of $q_{2}-q_{1}$ and $q_{1}$ that $q_{2}(a)-q_{1}(a), q_{2}(b)-q_{1}(b) \geq$ $q_{2}(13)-q_{1}(13)=\frac{5}{3}$, and $q_{1}(a)+q_{1}(b)-1 \leq q_{1}(13)+q_{1}(13)-1=-\frac{7}{3}$. So

$$
\varrho_{2}^{2}(\theta) \in\left\{[(x, 1, y)]_{\sim}: x, y \leq-\frac{5}{7}\right\} \subset B_{3} .
$$

Case (ii): We have $\varrho_{2}(\theta)=[(a, 0, b)] \sim$ for some $a>0, b>0$. So $\varrho_{2}(\theta) \notin B_{1}$. Moreover, by (4.10),

$\varrho_{2}^{2}(\theta)=\varrho_{2}\left([(a, 0, b)]_{\sim}\right)=[(a,-a-b, b)]_{\sim}=[(-a /(a+b), 1,-b /(a+b))]_{\sim} \in B_{3}$.

Case (iii): Simply observe that $\varrho_{2}(\theta) \in\left\{[(x, 1, y)]_{\sim}: x<0, y<0\right\} \subset B_{3}$.

$2^{\circ}$. Assume $\theta=[(x, 1, y)]_{\sim} \in B_{2}$. We show that $\varrho_{2}(\theta) \in L$. Then, since $L \cap B_{2}=\emptyset$ and, by Lemma 6.2, $L$ is invariant under $\varrho_{2}$, we conclude that $\varrho_{2}^{n}(\theta) \notin B_{2}$ for all $n>0$.

When $x, y \geq-3$,

$$
\frac{q_{2}(x)-q_{1}(x)}{q_{1}(x)+q_{1}(y)-1}, \frac{q_{2}(y)-q_{1}(y)}{q_{1}(x)+q_{1}(y)-1} \geq \frac{q_{2}(-3)-q_{1}(-3)}{q_{1}(-3)+q_{1}(-3)-1}=-\frac{1}{11} .
$$

On the other hand, $B_{2} \subset B_{3}$, so Lemma 6.2 implies that $\varrho_{2}\left(B_{2}\right) \subset B_{3}$. Combined with (6.2), we have $\varrho_{2}\left(B_{2}\right) \subset B_{3} \cap\left\{[x, 1, y] \sim: x, y \geq-\frac{1}{11}\right\}=L$.

\subsection{Distribution of 5}

One can verify [S1]-[S4] in a brute-force manner: formulas (4.2) suggest dividing the $(x, y)$ plane into 121 rectangles $I \times J$ (see Figure 3) so that $\max _{(x, y) \in I \times J} \varsigma(x, y)$ can be determined using bivariate calculus after verifying certain inequalities. In order to avoid checking the hundreds of inequalities needed in this brute-force approach, we observe that $S_{Q}$ and $S_{Q_{L}}$ share certain qualitative similarities, despite their quantitative difference.

If $\varsigma_{L}: \mathbb{P}\left(\mathbb{R}^{3}\right) \rightarrow[0, \infty)$ is defined similarly to $\varsigma$ but with $S_{Q}^{[1]}$ replaced by the linear $S_{Q_{L}}^{[1]}$, then if $(x, y) \neq(1,1)$,

$$
\varsigma_{L}([x, 1, y] \sim)=\frac{\max \left(D_{L}(x, y), D_{L}(y, x)\right)}{\max (|x-1|,|y-1|)}
$$

where $D_{L}(x, y)=\frac{1}{9} \max (|x-1|,|2(x-1)+(y-1)|)$. One can see either directly from the above expression or, more fundamentally, from the existence of $S_{Q_{L}}^{[2]}$ (see (2.18)) that if $s \neq 0$ and $|c| \leq 1$, then

$$
\varsigma_{L}\left([s+1,1, c s+1]_{\sim}\right)=\frac{2+c}{9} .
$$




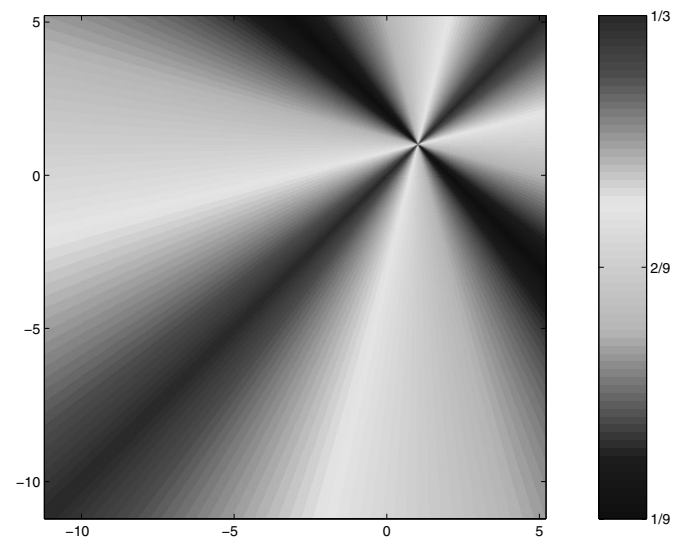

Fig. 5. "Linear Shrinking Factor" $\varsigma_{L}(x, y)$, see (6.4) and compare with Figure 3(a).

In particular, $\varsigma_{L}$ is: (i) constant in $s$; and (ii) increases from $\frac{1}{9}$ to $\frac{1}{3}$ as $c$ increases from -1 to 1 . For $\varsigma$, nonlinearity present in $S_{Q}$ spoils these properties, but we still have

Lemma 6.3. For any $s \in \mathbb{R}$ :

(i) $\varsigma([s+1,1, c s+1] \sim)$ is increasing in $c$ when $c \in[0,1]$;

(ii) $\varsigma([s+1,1, c s+1] \sim) \leq \varsigma([s+1,1,1] \sim)$ for $c \in[-1,0]$.

(It is not the case that $\varsigma([s+1,1, c s+1] \sim)$ is increasing on $c \in[-1,0]$ for all $s$.

Proof. It follows from (4.12) that

$$
\varsigma\left([s+1,1, c s+1]_{\sim}\right)= \begin{cases}\frac{\max (D(s+1, c s+1), D(c s+1, s+1))}{\max (|s|,|c s|)}, & \text { if } s \neq 0, \\ 0, & \text { if } s=0,\end{cases}
$$

where $D$ is given by (4.13).

We assume $s \neq 0$, otherwise the result is obviously true.

Since $|c| \leq 1, \max (|s|,|c s|)=|s|$.

To prove (i) it suffices to show that for a fixed $s$, both $D(s+1, c s+1)$ and $D(c s+1, s+1)$ are increasing in $c$ when $c \in[0,1]$. By (4.13), the latter is equivalent to proving that the absolute values of

$$
\begin{aligned}
E_{0}(s) & :=q_{1}(s+1)-2 q_{2}(s+1)+q_{3}(s+1), \\
E_{1}(c, s) & :=2 q_{1}(s+1)-q_{2}(s+1)+q_{1}(c s+1)-1, \\
E_{2}(c, s) & :=q_{1}(c s+1)-2 q_{2}(c s+1)+q_{3}(c s+1), \\
E_{3}(c, s) & :=2 q_{1}(c s+1)-q_{2}(c s+1)+q_{1}(s+1)-1,
\end{aligned}
$$

are increasing on $c \in[0,1]$ for any fixed $s$.

There is nothing to prove for $E_{0}$ as it is independent of $c$. We present the proof for $E_{1}$; those for $E_{2}$ and $E_{3}$ are completely analogous. Note that, by Lemma 6.1, $E_{i}$ is monotone 
on $c \in \mathbb{R}$, so the question basically boils down to whether monotonicity is spoiled by taking absolute value. Again, by Lemma 6.1,

$$
E_{1}(c, s)\left\{\begin{array}{l}
\leq E_{1}(0,0), \quad \text { when } c \geq 0, s>0, \\
\geq E_{1}(0,0), \quad \text { when } c \geq 0, s<0,
\end{array}\right\}=0 .
$$

Thus $\left|E_{1}(c, s)\right|=-\operatorname{sign}(s) E_{1}(c, s), \forall c>0, s \in \mathbb{R}$. Consequently, $\forall s \in \mathbb{R},\left|E_{1}(\cdot, s)\right|$ is increasing on the positive real axis, and on $[0,1]$ in particular.

Proving (ii) amounts to establishing the inequality

(6.5) $\max (D(s+1, c s+1), D(c s+1, s+1)) \leq \max (D(s+1,1), D(1, s+1))$,

$$
\forall c \in[-1,0], s \in \mathbb{R} \text {. }
$$

Note that the left-hand side of (6.5) is the maximum of four terms:

$$
\left|E_{0}(s)\right|=\left|E_{2}(1, s)\right|,\left|E_{i}(c, s)\right|, \quad i=1,2,3,
$$

whereas the right-hand side is the maximum of the absolute values of the following three (four to begin with, but one calculates to zero) terms:

$$
\begin{aligned}
E_{0}(s) & =E_{2}(1, s), \\
E_{1}(0, s) & =2 q_{1}(s+1)-q_{2}(s+1)+q_{1}(1)-1=2 q_{1}(s+1)-q_{2}(s+1)-\frac{1}{3}, \\
E_{2}(0, s) & =q_{1}(1)-2 q_{2}(1)+q_{3}(1)=0, \\
E_{3}(0, s) & =2 q_{1}(1)-q_{2}(1)+q_{1}(s+1)-1=q_{1}(s+1)-\frac{2}{3} .
\end{aligned}
$$

So both sides of (6.5) have the term $\left|E_{2}(1, s)\right|$ in common, thus (6.5) is proved after we establish

$$
\begin{aligned}
\forall c \in[-1,0], \quad s \in \mathbb{R}, \quad\left|E_{1}(c, s)\right| \leq\left|E_{1}(0, s)\right|, \\
\left|E_{2}(c, s)\right| \leq\left|E_{1}(0, s)\right|, \\
\left|E_{3}(c, s)\right| \leq \max \left\{\left|E_{1}(0, s)\right|,\left|E_{3}(0, s)\right|\right\} .
\end{aligned}
$$

These three inequalities can be proved in essentially the same manner.

By the monotonicity of $E_{1}(\cdot, s)$ (see Lemma 6.1) and by verifying that $E_{1}(-1, \cdot)$ is strictly decreasing (using (4.2)), we have

$$
\begin{aligned}
& 0=E_{1}(-1,0)>E_{1}(-1, s) \geq E_{1}(c, s) \geq E_{1}(0, s), \quad \text { when } c \in[-1,0], \quad s>0, \\
& 0=E_{1}(-1,0)<E_{1}(-1, s) \leq E_{1}(c, s) \leq E_{1}(0, s), \quad \text { when } c \in[-1,0], \quad s<0 .
\end{aligned}
$$

Hence (6.10) is proved.

Similarly, it follows from the monotonicity of $E_{2}(\cdot, s)$ and the fact

$$
E_{2}(-1, s) \begin{cases}\geq E_{1}(0, s) & \text { when } s>0 \\ \leq E_{1}(0, s) & \text { when } s<0\end{cases}
$$

(verifiable by (4.2)) that

$$
\begin{aligned}
& 0=E_{2}(0, s) \geq E_{2}(c, s) \geq E_{2}(-1, s) \geq E_{1}(0, s), \quad \text { when } c \in[-1,0], \quad s>0, \\
& 0=E_{2}(0, s) \leq E_{2}(c, s) \leq E_{2}(-1, s) \leq E_{1}(0, s), \quad \text { when } c \in[-1,0], \quad s<0 .
\end{aligned}
$$

Therefore (6.11) is proved. 
Finally, (6.12) can be established by proving

$$
\begin{aligned}
& E_{3}(0, s) \leq E_{3}(c, s) \leq E_{3}(-1, s) \leq-E_{1}(0, s), \quad \text { when } c \in[-1,0], \quad s>0, \\
& E_{3}(0, s) \geq E_{3}(c, s) \geq E_{3}(-1, s) \geq-E_{1}(0, s), \quad \text { when } c \in[-1,0], \quad s<0 .
\end{aligned}
$$

The first two inequalities in each chain are just consequences of the monotonicity of $E_{3}(\cdot, s)$ and the last inequality in each chain can be verified by (4.2).

Proof of [S4]. Let $G_{0}:=\left\{[(x, 0, y)]_{\sim}: x y \leq 0\right\}$ and let $G_{1}:=\left\{[(x, 1, y)]_{\sim}\right.$ : $(x-1)(y-1) \leq 0\}$, then

$$
\begin{aligned}
G & =\left\{\left[\left(a_{1}, a_{2}, a_{3}\right)\right]_{\sim} \in \mathbb{P}\left(\mathbb{R}^{3}\right):\left(a_{1}-a_{2}\right)\left(a_{3}-a_{2}\right) \leq 0\right\} \\
& =G_{0} \cup G_{1} .
\end{aligned}
$$

When $\theta=[(x, 0, y)]_{\sim} \in G_{0}$, we have $x y \leq 0$. So $|2 x+y| \leq \max (2|x|,|y|)$ and $\mid x+$ $2 y \mid \leq \max (|x|, 2|y|)$. It follows that $\max (|x|,|2 x+y|,|x+2 y|,|y|) \leq 2 \max (|x|,|y|)$. So, by (4.12), $\varsigma(\theta) \leq \frac{2}{9}$. When $\theta=[(x, 1, y)]_{\sim} \in G_{1}$, we can assume $(x, y) \neq(1,1)$ as $\varsigma(1,1)=0$. Notice also the symmetry property $\varsigma(x, y)=\varsigma(y, x)$. So we have

$$
\begin{aligned}
& \max _{\theta \in G_{1}} \varsigma(\theta)=\max _{\substack{(x-1)(y-1) \leq 0 \\
(x, y) \neq(1,1)}} \varsigma(x, y)=\max _{\substack{-1 \leq \frac{y-1}{x-1} \\
x \neq 1}} \varsigma(x, y) \\
& =\max _{\substack{c \in[-1,0] \\
s \in \mathbb{R} \\
s \neq 0}} \varsigma(s+1, c s+1) \stackrel{\text { Lemma }}{=}{ }^{3(\mathrm{ii})} \max _{\substack{s \in \mathbb{R} \\
s \neq 0}} \varsigma(s+1,1) .
\end{aligned}
$$

From (4.12) and (4.13), for $s \neq 0$,

$$
\begin{aligned}
& \varsigma(s+1,1) \\
& =\frac{\max (D(s+1,1), D(1, s+1))}{|s|} \\
& =\frac{\max \left(\left|q_{1}(s+1)-2 q_{2}(s+1)+q_{3}(s+1)\right|,\left|2 q_{1}(s+1)-q_{2}(s+1)-\frac{1}{3}\right|,\left|q_{1}(s+1)-\frac{2}{3}\right|\right)}{|s|} .
\end{aligned}
$$

With computational details omitted, one can show using (4.2) that the one-dimensional maximization problem $\sup _{s \neq 0} \varsigma(s+1,1)$ has the solution $\frac{34}{135}$ and the unique maximizer $s=-2$. Summarizing,

$$
\max _{\theta \in G} \varsigma(\theta)=\max \left(\max _{\theta \in G_{0}} \varsigma(\theta), \max _{\theta \in G_{1}} \varsigma(\theta)\right)=\max \left(\frac{2}{9}, \frac{34}{135}\right)=\frac{34}{135}=\varsigma(-1,1)=\varsigma(1,-1) .
$$

Proof of [S1]. If $\theta=[(x, 0, y)]_{\sim}$, then, by $(4.12), \varsigma(\theta) \leq \frac{1}{3}$.

So $\mathcal{B}=\left\{\theta \in \mathbb{P}\left(\mathbb{R}^{3}\right): \varsigma(\theta)>\frac{1}{3}\right\} \subset\left\{[(x, 1, y)]_{\sim}:(x, y) \neq(1,1)\right\}$. Since $\max _{\theta \in G} \zeta(\theta)=\frac{34}{135}<\frac{1}{3}$, we have $\mathcal{B} \subset\left\{[(x, 1, y)]_{\sim}:(x-1)(y-1)>0\right\}$.

Suppose $\theta=[(x, 1, y)]_{\sim} \in \mathcal{B}$, i.e., $\varsigma(x, y)>\frac{1}{3}$, then $(x-1)(y-1)>0$. Let $x=s+1$ and $c=(y-1) /(x-1)$, then $y=c s+1$ and $s \neq 0, c>0$. 
It suffices to prove:

(1) if $s>0$ and $c \in(0,1]$, then $\theta \in\left\{[(x, 1, y)]_{\sim}:(x, y) \in\left[\frac{7}{3}, 5\right]^{2}, y \leq x\right\}$;

(2) if $s>0$ and $c>1$, then $\theta \in\left\{[(x, 1, y)] \sim:(x, y) \in\left[\frac{7}{3}, 5\right]^{2}, y>x\right\}$;

(3) if $s<0$ and $c \in(0,1]$, then $\theta \in\left\{[(x, 1, y)]_{\sim}:(x, y) \in\left[-3,-\frac{1}{11}\right)^{2}, y \geq x\right\}$;

(4) if $s<0$ and $c>1$, then $\theta \in\left\{[(x, 1, y)] \sim:(x, y) \in\left[-3,-\frac{1}{11}\right)^{2}, y<x\right\}$.

We first prove (1). Assume $s>0$ and $c \in(0,1]$, then it follows from Lemma 6.3(i) that $\varsigma(x, y)=\varsigma(s+1, c s+1) \leq \varsigma(s+1, s+1)$. So

$$
\varsigma(s+1, s+1)>\frac{1}{3} .
$$

Consider

$$
\varsigma(t, t)=\frac{D(t, t)}{|t-1|}=\frac{\max \left(\left|q_{1}(t)-2 q_{2}(t)+q_{3}(t)\right|,\left|3 q_{1}(t)-q_{2}(t)-1\right|\right)}{|t-1|} .
$$

By applying Lemma 6.1, it is easy to show

$$
\varsigma(t, t)=\frac{1+q_{2}(t)-3 q_{1}(t)}{t-1} .
$$

Combining with (4.2), we can easily prove that $\varsigma(t, t)>\frac{1}{3}$ implies $t \in\left[-3,-\frac{1}{11}\right) \cup$ $\left[\frac{7}{3}, 5\right]$. Combining this and (6.13) would lead to $x=s+1 \in\left[\frac{7}{3}, 5\right]$. So $y=c s+1 \leq$ $s+1=x \leq 5$.

Since $x \in\left[\frac{7}{3}, 5\right]$, it follows from (4.2) that $q_{1}(x)=\frac{59}{27}+\frac{7}{27} x-\frac{8}{27} \sqrt{x^{2}+16 x+16}$, $q_{2}(x)=1, q_{3}(x)=\frac{11}{27}+\frac{1}{27} x+\frac{4}{27} \sqrt{x^{2}+16 x+16}$. By applying these formulas, it can be proved by elementary calculus that $\varsigma\left(x, \frac{7}{3}\right) \leq \frac{1}{3}$. It follows from Lemma 6.3(i) and $\varsigma(x, y)>\frac{1}{3}$ that $y>\frac{7}{3}$.

Therefore $(x, y) \in\left[\frac{7}{3}, 5\right]^{2}$ and $y \leq x$, i.e., (1) is proved.

Similarly, we can prove (2), (3), (4).

Proof of [S2]. Let $\theta=[(x, 1, y)] \sim \in B_{1}$, i.e., $(x, y) \in\left[\frac{7}{3}, 5\right]^{2}$. Since $\varsigma(x, y)=$ $\varsigma(y, x)$, it follows from Lemma 6.3(i) and (6.14) that

$$
\max _{(x, y) \in\left[\frac{7}{3}, 5\right]^{2}} \varsigma(x, y)=\max _{\substack{(x, y) \in\left[\frac{7}{3}, 5\right]^{2} \\ y \leq x}} \varsigma(x, y)=\max _{x \in\left[\frac{7}{3}, 5\right]} \varsigma(x, x)=\max _{x \in\left[\frac{7}{3}, 5\right]} \frac{1+q_{2}(x)-3 q_{1}(x)}{x-1} .
$$

Applying (4.2), it is easy to show

$$
\max _{x \in\left[\frac{7}{3}, 5\right]} \frac{1+q_{2}(x)-3 q_{1}(x)}{x-1}=\frac{1+q_{2}\left(\frac{16}{5}\right)-3 q_{1}\left(\frac{16}{5}\right)}{\frac{16}{5}-1}=\frac{35}{99} .
$$

Therefore $\max _{\theta \in B_{1}} \varsigma(\theta)=\varsigma\left(\frac{16}{5}, \frac{16}{5}\right)=\frac{35}{99}$.

A similar calculation establishes [S3]. 


\subsection{Derivative of M-Estimator}

Here we only try to address Gâteaux differentiability, i.e., the first part of question (Q) in Section 5. This question appears to be rather technical even in the median case $\sigma=|\cdot|$. If we restrict the domain of $m_{|\cdot|}$ to the finite-dimensional $X=\Pi_{n}$ (any $n \geq 1$ ), then by [6, Prop. 3.1] the linear functional

$$
\bar{m}_{|\cdot|}: C(I) \rightarrow \mathbb{R}, \quad f \mapsto f(\text { midpoint of } I),
$$

when restricted to $X$, is the derivative of the median functional $m_{|\cdot|}: X \rightarrow \mathbb{R}$ at any strictly monotone $l \in \Pi_{n}$. (Note: From the mere fact that $m_{|\cdot|}$ is both homogeneous and nonlinear, $m_{|\cdot|}$ cannot be differentiable at the zero function; since $m_{|\cdot|}$ is also offset invariant, it cannot be differentiable at any constant function.) The same conclusion also holds if $X=C^{1}(I)$, this is because for $f, g \in C^{1}(I)$, if $f$ is strictly monotone, then so is $f+\varepsilon g$ for a small enough $\varepsilon$. The same is not true if we only know that $f$ and $g$ are continuous, so the same argument cannot be used to prove that the same conclusion holds when $X$ is enlarged back to $C(I)$.

Below, we formally calculate the derivative of $m_{\sigma}$ at a strictly monotone function $l$, eventually arriving at the "answer" (6.20)-(6.21).

We are interested in the derivative

$$
D m_{\sigma}(f ; l):=\lim _{\varepsilon \rightarrow 0} \frac{m_{\sigma}(l+\varepsilon f)-m_{\sigma}(l)}{\varepsilon},
$$

for $f \in C(I)$. The numerator in the divided difference equals

$$
\begin{aligned}
\underset{m}{\arg \min } \int_{I} \sigma(l(x)+\varepsilon f(x)-m) d x-m_{\sigma}(l) \\
\quad=\underset{m}{\operatorname{root}} \int_{I} \sigma^{\prime}(l(x)+\varepsilon f(x)-m) d x-m_{\sigma}(l) .
\end{aligned}
$$

For small $\varepsilon$, the root $m$ involved in the right-hand side of (6.17) is close to $m_{\sigma}(l)$, so by the Taylor expansion:

$$
\begin{aligned}
& \sigma^{\prime}(l(x)+\varepsilon f(x)-m) \\
& \quad \approx \sigma^{\prime}\left(l(x)-m_{\sigma}(l)\right)+\sigma^{\prime \prime}\left(l(x)-m_{\sigma}(l)\right)\left(\varepsilon f(x)-m+m_{\sigma}(l)\right), \quad \varepsilon \text { small. }
\end{aligned}
$$

As such, we approximate the nonlinear scalar equation

$$
N(m):=\int_{I}(\text { left-hand side of (6.18)) } d x=0
$$

by the linear scalar equation

$$
\bar{N}(m):=\int_{I}(\text { right-hand side of (6.18)) } d x=0,
$$

the solution of the latter can be easily found to be

$$
m=\frac{\varepsilon \int_{I} \sigma^{\prime \prime}\left(l(x)-m_{\sigma}(l)\right) f(x) d x}{\int_{I} \sigma^{\prime \prime}\left(l(x)-m_{\sigma}(l)\right) d x}+m_{\sigma}(l) .
$$


Hence, for small $\varepsilon$, the divided quotient in (6.16) is very well approximated by

$$
\frac{\int_{I} \sigma^{\prime \prime}\left(l(x)-m_{\sigma}(l)\right) f(x) d x}{\int_{I} \sigma^{\prime \prime}\left(l(x)-m_{\sigma}(l)\right) d x}
$$

this must be the sought-for derivative, well, if the derivative actually exists and the integrals in (6.19) actually make sense.

If the Gâteaux derivative of $m_{\sigma}$ at $l$ exists then, by definition, it is a bounded linear functional on $C(I)$. In fact, since $m_{\sigma}(f) \geq 0$ for $f \geq 0$, this bounded linear functional, if exists, must be a positive linear functional on $C(I)$. In light of the Riesz representation theorem for bounded linear functionals on $C(I)$, we define the strictly monotone functions

$$
\xi(x):=\int_{a}^{x} \frac{d \sigma^{\prime}\left(l(t)-m_{\sigma}(l)\right)}{l^{\prime}(t)}, \quad \alpha(x):=\frac{\xi(x)}{\int_{I} d \xi(x)},
$$

then we can rewrite $(6.19)$ as

$$
D m_{\sigma}(f ; l):=\int_{I} f(x) d \alpha(x)
$$

All the integrals here are now interpreted in the Riemann-Stieltjes sense. The punchline of rewriting (6.19) in this form is that now, as long as $l$ is, say, $C^{1}$ and $l^{\prime}>0$ (or $l^{\prime}<0$ ) and $\sigma$ is a convex function, then all the derivatives and integrals in (6.20)-(6.21) are well defined and (6.21) indeed defines a continuous positive linear functional. For example, when $\sigma=|\cdot|, \sigma^{\prime}=\operatorname{sign}(\cdot)$ and (6.21) is well defined in the Riemann-Stieltjes sense and indeed recovers (6.15), whereas (6.19) has no meaning at all.

Despite the lack of rigor in our heuristical solution to $(\mathrm{Q})$, we now have the positive linear functional $\operatorname{Dm}_{\sigma}(\cdot ; l)$ rigorously defined in (6.20)-(6.21).

Acknowledgments. The work of this research was partially supported by an NSF CAREER Award (CCR 9984501). The authors would like to thank two referees for constructive comments on the original version of the paper, which have led to an improvement of the presentation.

\section{G. Xie}

Department of Mathematical Sciences

Rensselaer Polytechnic Institute

Troy, NY 12180

USA

xieg@rpi.edu
T. P.-Y. Yu

Department of Mathematical Sciences

Rensselaer Polytechnic Institute

Troy, NY 12180

USA

yut@rpi.edu 